\title{
TRAVELING WAVE SOLUTIONS TO A COUPLED SYSTEM OF SPATIALLY DISCRETE NAGUMO EQUATIONS*
}

\author{
MICHAEL D. BATEMAN ${ }^{\dagger}$ AND ERIK S. VAN VLECK ${ }^{\dagger}$
}

\begin{abstract}
We consider a coupled system of discrete Nagumo equations and derive traveling wave solutions to this system using McKean's caricature of the cubic. A certain form of this system is used to model ephaptic coupling between pairs of nerve axons. We study the difference $g(c)=a_{1}-a_{2}$ between the detuning parameters $a_{i}$ that is required to make both waves move at the same speed $c$. Of particular interest is the effect of a coupling parameter $\alpha$ and an "alignment" parameter $A$ on the function $g$. Numerical investigation indicates that for fixed $A$, there exists a time delay value $\beta$ that results in $g=0$, and for large enough wave speeds, multiple such $\beta$ values exist. Also, numerical results indicate that the perturbation of $\alpha$ away from zero will yield additional solutions with positive wave speed when $A=\frac{1}{2}$. We employ both analytical and numerical results to demonstrate our claims.
\end{abstract}

Key words. discrete Nagumo equations, ephaptic coupling, traveling waves

AMS subject classifications. 35K57, 74N99

DOI. $10.1137 / 050624352$

1. Introduction. In myelinated nerve axons, transmembrane ion flow occurs only at the spatially periodic nodes of Ranvier, and the activity at these nodes may affect the activity at nodes of neighboring fibers. This so-called ephaptic coupling is an electrical effect that causes neighboring fibers to interact and possibly synchronize with each other. Accompanying the problem of ephaptic coupling is the issue of the relative positioning of the nodes of Ranvier on the different fibers. That is, given two parallel nerve fibers, the nodes on one fiber may or may not align perfectly with the nodes on the other fiber.

Our contribution in this paper is to derive a solution to a system that models these phenomena and use this model to show that coupling decreases the size of the range of propagation failure when the nodes of Ranvier are staggered, but that coupling increases the size of this range when the nodes are perfectly aligned. To do this, we consider a system of two myelinated nerve axons coupled ephaptically. In particular, our goal is to study the effect of this coupling and the effect of nonalignment on the propagation of action potentials. Different types of coupling between fibers are possible. In [2], Binczak, Eilbeck, and Scott model "saltatory" conduction present in these myelinated neurons with equations used to govern the behavior of electrical circuits and introduce the effect of ephaptic coupling between two myelinated neurons. In [1] a different type of coupling, called "ohmetric" coupling, is considered and it is shown that the introduction of this kind of coupling causes waves on two adjacent myelinated axons to match speeds with each other. Earlier work of Keener [13] and Bose and Jones $[4,5]$ addressed the issue of ohmetric coupling.

The strength of the ephaptic coupling $\alpha$ depends on the electrical resistance $R_{\text {int }}$ inside the axons (assumed to be the same for both axons) and the resistance $R_{o}$ of the medium between the axons. We must also consider the positioning of the axons

\footnotetext{
${ }^{*}$ Received by the editors February 14, 2005; accepted for publication (in revised form) October 3, 2005; published electronically March 3, 2006. This work was supported in part by NSF grants DMS-0139824 and DMS-0513438.

http://www.siam.org/journals/siap/66-3/62435.html

${ }^{\dagger}$ Department of Mathematics, University of Kansas, Lawrence, KS 66045 (mbat@ku.edu, evanvleck@math.ku.edu).
} 


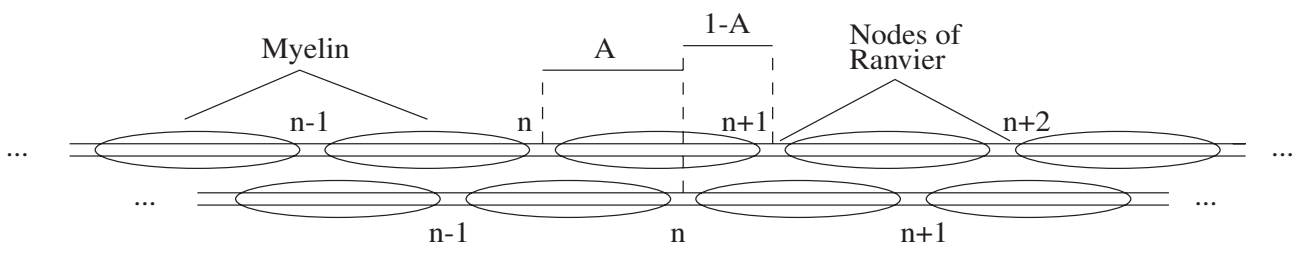

FIG. 1. Diagram of two myelinated nerve axons.

relative to each other: here we assume that they are parallel, that the nodes on a given fiber are evenly spaced, and that the distance between nodes is the same on both fibers, but we allow the nodes of Ranvier to line up or not line up, as shown in Figure 1. That is, we will introduce an alignment parameter $A$ that will reflect the positioning of the nodes on one fiber relative to those on the other. We will also introduce a parameter $\beta$ that will act as a time delay on one of the fibers. Specifically, we consider a system of differential equations on two one-dimensional lattices coupled together. Study of the uncoupled case $[6,11,10,14]$ provides an idea of the kind of behavior to expect from the coupled system and also serves as a precedent to which our results may be compared.

We consider the system

$$
\begin{aligned}
\dot{V}_{n}^{(1)}+f_{1}\left(V_{n}^{(1)}\right)=\frac{1}{1-\alpha^{2}}[ & D_{1}\left(\bar{L} V^{(1)}\right)_{n}-\alpha D_{2}\left(\bar{L} V^{(2)}\right)_{n}-\alpha A\left(\bar{N} V^{(2)}\right)_{n} \\
& \left.-\alpha^{2} A\left(\bar{N} V^{(1)}\right)_{n+1}\right] \\
\dot{V}_{n}^{(2)}+f_{2}\left(V_{n}^{(2)}\right)=\frac{1}{1-\alpha^{2}}[ & -\alpha D_{1}\left(\bar{L} V^{(1)}\right)_{n}+D_{2}\left(\bar{L} V^{(2)}\right)_{n}+\alpha^{2} A\left(\bar{N} V^{(2)}\right)_{n} \\
& \left.+\alpha A\left(\bar{N} V^{(1)}\right)_{n+1}\right]
\end{aligned}
$$

where $(\bar{L} V)_{n}=V_{n+1}-2 V_{n}+V_{n-1},\left(\bar{N} V^{(i)}\right)_{n}=\left(\dot{V^{(i)}}{ }_{n-1}-\dot{V^{(i)}}{ }_{n}\right)+\left(f_{i}\left(V_{n-1}^{(i)}\right)-\right.$ $\left.f_{i}\left(V_{n}^{(i)}\right)\right), D_{1}, D_{2}>0$ are diffusion coefficients, $A \in[0,1)$ is the alignment parameter, and $\alpha \in[0,1)$ is the coupling coefficient. When $\alpha=0$, the ephaptic coupling is completely turned off, and increasing $\alpha$ increases the strength of the coupling. When $A=0$, the nodes are in perfect alignment; setting $A=\frac{1}{2}$, for example, staggers the nodes so that the $n$th node on one fiber is equidistant from the $n$th and $(n+1)$ th nodes on the other fiber. The nonlinearities $f_{i}: \mathbb{R} \rightarrow \mathbb{R}$ are often cubics of the form $f_{i}(u)=u\left(u-a_{i}\right)(u-1)$, where the $a_{i} \in(0,1)$ are "detuning" parameters, and the quantities $V_{n}^{(i)}$ represent the ionic flow through the membrane at the $n$th node of the respective fibers. However, to facilitate the construction of a solution, we consider an idealized, piecewise linear $f_{i}$ known as McKean's caricature of the cubic (see $[15,17,12,22,23,6,11,8,9])$. When considering one fiber, it is only necessary to think of the detuning parameter $a \in\left(0, \frac{1}{2}\right)$ because of symmetry in the relationship between the detuning parameter and the wave speed. A priori, we do not know if the same is true when considering two fibers. However, we will see in Theorem 4.4 and Corollary 4.5 that certain symmetries do hold in the coupled problem.

In general, the wave speed $c$ depends on all the other parameters in the problem. Here, however, we approach the issue from a different angle: we fix all parameters except the detuning parameters $a_{i}$ and assume that the wave speed $c$ is the same for 
both fibers. This way we "solve," in a sense, for the detuning parameters. Instead of fixing the detuning parameters, and studying how they affect the wave speeds, we demand that the wave speeds be the same and determine which pairs of detuning parameters give us this effect.

Although our particular model is motivated by this neurological application, similar models may also be used to study action potentials in cardiac cells, among other things (see $[3,16,20,21])$. The authors in [1] also mention the possible application of coupled two-dimensional lattices to the study of image processing. For this reason, we consider a more general system and allow the parameters to range over values that may not be physically reasonable for our particular application.

This paper is organized as follows: we start by using a piecewise linear nonlinearity $f$ to derive candidate solutions using a Fourier transform method. To make this process easier, we make a key assumption about the shape of our solutions - this assumption will be verified after we have obtained the candidate solutions. The details of the construction have been left to an appendix. After proving the existence of traveling backs, we look at some properties held by these solutions. We investigate the relationship between the wave speed $c$ and the detuning parameters $a_{i}$, as well as the effects of the coupling coefficient $\alpha$ and the alignment parameter $A$ on this system. We have interspersed some numerical computations throughout the paper to illustrate the analytical results and provide insight into the problem.

1.1. Derivation of the system. The system considered here is a version of the model used in [18] (see pp. 177-183) and [2]. The variables used here are as follows: $I_{n}$ is the mesh current, a term used to analyze circuits using Kirchhoff's equations; $I_{i o n, n}$ is a cubic modeling the ionic current (taking into consideration both sodium and potassium); $V_{n}^{(i)}$ is the voltage across a node of Ranvier, and $V_{b}$ is a constant representing the Nernst potential for sodium ions; $R_{i, j}$ is the resistance inside fiber $j$, $R_{o}$ is the resistance outside the axons, and $R_{f}$ is a constant representing the internodal resistance, and is on the same order as $R_{i, j}$; and $C$ is the capacitance of a node plus the capacitance of the adjacent internodal myelin sheath.

Using the circuit diagrams presented in [2] and [18], we sum the voltages around the mesh and set the result to zero. Note that here we will use $A$ to represent the amount that fiber 1 leads fiber 2 . This gives us

$$
\begin{aligned}
& v_{n}^{(1)}-v_{n+1}^{(1)}=\left(R_{i, 1}+R_{o}\right) I_{n}^{(1)}+R_{o}\left(A I_{n-1}^{(2)}+(1-A) I_{n}^{(2)}\right), \\
& v_{n}^{(2)}-v_{n+1}^{(2)}=\left(R_{i, 2}+R_{o}\right) I_{n}^{(2)}+R_{o}\left(A I_{n+1}^{(1)}+(1-A) I_{n}^{(1)}\right) .
\end{aligned}
$$

In addition, we have the following relationship between the mesh currents and the voltages:

$$
I_{n-1}^{(j)}-I_{n}^{(j)}=C \frac{d v_{n}^{(j)}}{d t}+I_{i o n, n}^{(j)}, \quad j=1,2 .
$$

Now we multiply (1.2) by $\frac{R_{f}}{V_{b}\left(R_{i, j}+R_{o}\right)}$ and (1.3) by $\frac{R_{f}}{V_{b}}$. Using the notation

$$
V_{n}^{(j)}=\frac{v_{n}^{(j)}}{V_{b}}, \quad i_{n}^{(j)}=\frac{R_{f} I_{n}^{(j)}}{V_{b}}, \quad D_{j}=\frac{R_{f}}{R_{i, j}+R_{o}}, \quad \text { and } \alpha_{j}=\frac{R_{o}}{R_{i, j}+R_{o}},
$$

we arrive at

$$
\begin{aligned}
& D_{1}\left(V_{n}^{(1)}-V_{n+1}^{(1)}\right)=i_{n}^{(1)}+\alpha\left(A i_{n-1}^{(2)}+(1-A) i_{n}^{(2)}\right), \\
& D_{2}\left(V_{n}^{(2)}-V_{n+1}^{(2)}\right)=i_{n}^{(2)}+\alpha\left(A i_{n+1}^{(1)}+(1-A) i_{n}^{(1)}\right),
\end{aligned}
$$




$$
\begin{aligned}
& i_{n-1}^{(1)}-i_{n}^{(1)}=R_{f} C \dot{V}_{n}^{(1)}+f_{1}\left(V_{n}^{(1)}\right), \\
& i_{n-1}^{(2)}-i_{n}^{(2)}=R_{f} C \dot{V}_{n}^{(2)}+f_{2}\left(V_{n}^{(2)}\right) .
\end{aligned}
$$

As noted in [2], experimental results indicate that $R_{o} \ll R_{i, j} \approx R_{f}$, so we take $\alpha_{1}=\alpha_{2}$ but allow $D_{1} \neq D_{2}$.

Using (1.5) and (1.6), we have

$$
\left[\begin{array}{ll}
1 & \alpha \\
\alpha & 1
\end{array}\right]\left[\begin{array}{c}
i_{n}^{(1)} \\
i_{n}^{(2)}
\end{array}\right]=\left[\begin{array}{c}
X_{n}^{(1)} \\
X_{n}^{(2)}
\end{array}\right],
$$

where

$$
\begin{aligned}
& X_{n}^{(1)}=D_{1}\left(V_{n}^{(1)}-V_{n+1}^{(1)}\right)-\alpha A\left(R_{f} C \dot{V}_{n}^{(2)}+f\left(V_{n}^{(2)}\right)\right), \\
& X_{n}^{(2)}=D_{2}\left(V_{n}^{(2)}-V_{n+1}^{(2)}\right)+\alpha A\left(R_{f} C \dot{V}_{n+1}^{(1)}+f\left(V_{n+1}^{(1)}\right)\right)
\end{aligned}
$$

which gives us

$$
i_{n}^{(1)}=\frac{1}{1-\alpha^{2}}\left(X_{n}^{(1)}-\alpha X_{n}^{(2)}\right), \quad i_{n}^{(2)}=\frac{1}{1-\alpha^{2}}\left(X_{n}^{(2)}-\alpha X_{n}^{(1)}\right) .
$$

Substituting into (1.6), we have

$$
\begin{aligned}
R_{f} C \dot{V}_{n}^{(1)}+f_{1}\left(V_{n}^{(1)}\right) & =\frac{1}{1-\alpha^{2}}\left[\left(X_{n-1}^{(1)}-X_{n}^{(1)}\right)-\alpha\left(X_{n-1}^{(2)}-X_{n}^{(2)}\right)\right], \\
R_{f} C \dot{V}_{n}^{(2)}+f_{2}\left(V_{n}^{(2)}\right) & =\frac{1}{1-\alpha^{2}}\left[\left(X_{n-1}^{(2)}-X_{n}^{(2)}\right)-\alpha\left(X_{n-1}^{(1)}-X_{n}^{(1)}\right)\right] .
\end{aligned}
$$

Computing $X_{n-1}^{(j)}-X_{n}^{(j)}$ for $j=1,2$ and inserting the resulting expressions into (1.10) brings us to system (1.1). Note that we will take $R_{f} C=1$ since changing these parameters will only amount to a rescaling of the wave speed $c$ after we impose a traveling wave ansatz.

2. Construction of a solution. In the construction of our solution, the speed $c$ is assumed to be nonzero unless otherwise noted, and the nonlinearity $f_{i}$ will be the idealized cubic-like function

$$
f_{i}(u)=u-h\left(u-a_{i}\right),
$$

where $h$ denotes the Heaviside step function

$$
h(u)= \begin{cases}0 & \text { if } u<0 \\ {[0,1]} & \text { if } u=0 \\ 1 & \text { if } u>0\end{cases}
$$

Note that $h(u)$ is a set-valued function, evaluating to the interval $[0,1]$ when $u=0$ and evaluating to a singleton everywhere else. This results in the $f_{i}(u)$ being set-valued functions as well. Thus, (1.1) with $f_{i}$ given in (2.1) and (2.2) should be interpreted as a differential inclusion when $V_{n}^{(i)}=a_{i}$. 
After imposing the traveling wave ansatz $V_{n}^{(i)}(t)=\varphi_{i}(n-c t)$, and letting $\xi=$ $n-c t$, we have

$$
\begin{aligned}
-c \varphi_{1}^{\prime}(\xi)+f_{1}\left(\varphi_{1}(\xi)\right)=\frac{1}{1-\alpha^{2}}[ & D_{1}\left(L \varphi_{1}\right)(\xi)-\alpha D_{2}\left(L \varphi_{2}\right)(\xi) \\
& \left.-\alpha A\left(N \varphi_{2}\right)(\xi)-\alpha^{2} A\left(N \varphi_{1}\right)(\xi+1)\right], \\
-c \varphi_{2}^{\prime}(\xi)+f_{2}\left(\varphi_{2}(\xi)\right)=\frac{1}{1-\alpha^{2}}[ & -\alpha D_{1}\left(L \varphi_{1}\right)(\xi)+D_{2}\left(L \varphi_{2}\right)(\xi) \\
& \left.+\alpha^{2} A\left(N \varphi_{2}\right)(\xi)+\alpha A\left(N \varphi_{1}\right)(\xi+1)\right],
\end{aligned}
$$

where

$$
\begin{aligned}
(L \varphi)(\xi) & =\varphi(\xi+1)-2 \varphi(\xi)+\varphi(\xi-1) \\
(N \varphi)(\xi) & =-c\left[\varphi^{\prime}(\xi-1)-\varphi^{\prime}(\xi)\right]+[f(\varphi(\xi-1))-f(\varphi(\xi))] .
\end{aligned}
$$

It is natural to require the boundary conditions

$$
\varphi_{i}(-\infty)=0, \quad \varphi_{i}(+\infty)=1
$$

for $i=1,2$. To help construct solutions to this system, we will initially assume that each $\varphi_{i}$ satisfies $\varphi_{i}\left(\beta_{i}\right)=a_{i}$ for only one value, and we may assume one of these values to be zero by simply translating the argument. That is, we assume

$$
\varphi_{i}(\xi) \begin{cases}<a_{i} & \text { for } \quad \xi<\beta_{i}, \\ =a_{i} & \text { for } \quad \xi=\beta_{i} \\ >a_{i} & \text { for } \quad \xi>\beta_{i}\end{cases}
$$

where we will take $\beta_{1}=0$ and $\beta_{2}=\beta$. After we construct our candidate solutions, we will verify that they satisfy these assumptions.

With (2.6), we have that $h\left(\varphi_{i}(\xi)-a_{i}\right)=h\left(\xi-\beta_{i}\right)$, which gives us $f\left(\varphi_{i}(\xi)\right)=$ $\varphi_{i}(\xi)-h\left(\xi-\beta_{i}\right)$. Now the system $(2.3)$ becomes

$$
\begin{aligned}
-c \varphi_{1}^{\prime}(\xi)=\frac{1}{1-\alpha^{2}}[ & D_{1}\left(L \varphi_{1}\right)(\xi)-\alpha D_{2}\left(L \varphi_{2}\right)(\xi) \\
\left.-\alpha A\left(N \varphi_{2}\right)(\xi)-\alpha^{2} A\left(N \varphi_{1}\right)(\xi+1)\right]-\varphi_{1}(\xi)+h(\xi), & \\
-c \varphi_{2}^{\prime}(\xi)=\frac{1}{1-\alpha^{2}}[ & -\alpha D_{1}\left(L \varphi_{1}\right)(\xi)+D_{2}\left(L \varphi_{2}\right)(\xi) \\
& \left.+\alpha^{2} A\left(N \varphi_{2}\right)(\xi)+\alpha A\left(N \varphi_{1}\right)(\xi+1)\right]-\varphi_{2}(\xi)+h(\xi-\beta) .
\end{aligned}
$$

Notice the presence of several Heaviside functions in each equation of (2.7). Each instance of the Heaviside function results in a discontinuity of the first derivative of the functions $\varphi_{i}$. For example, we expect to have discontinuities in the first derivative of $\varphi_{1}$ at $\xi=0, \xi=1, \xi=\beta$, and $\xi=\beta+1$. Further, an appearance of $\varphi^{\prime}(\xi+1)$ in the first equation leads us to expect a discontinuity in the first derivative of $\varphi_{1}$ at $\xi=-1$. These discontinuities will be reflected in the solution we compute.

As outlined in Appendix A, candidate solutions to this system can be expressed as

$$
\varphi_{i}(\xi)=\frac{1}{2}+\frac{1}{2 \pi} \int_{0}^{\infty} \sum_{j \in M_{i}} F_{i, j}(s) \cos (s(\xi-j))+G_{i, j}(s) \frac{1}{s} \sin (s(\xi-j)) d s
$$


where $M_{i}=\left\{\beta_{i}, \beta_{i}+1, \beta_{i}-1, \beta_{i^{*}}, \beta_{i^{*}}+1\right\}, 1^{*}=2$ and $2^{*}=1$, and

$$
\begin{array}{rlrl}
F_{i, \beta_{i}}(s) & =\left[W(s)(1+2 Q)-R_{i^{*}}(s) Y(s)\right], \quad G_{i, \beta_{i}}(s) & =\left[X(s)(1+2 Q)-R_{i^{*}}(s) Z(s)\right], \\
F_{i, \beta_{i}+1}(s) & =-Q W(s), & G_{i, \beta_{i}+1}(s) & =-Q X(s), \\
F_{i, \beta_{i}-1}(s) & =-Q W(s), & G_{i, \beta_{i}-1}(s) & =-Q X(s), \\
F_{i, \beta_{i^{*}}}(s) & =-\alpha(1-A) R_{i^{*}}(s) Y(s), & G_{i, \beta_{i^{*}}}(s) & =-\alpha(1-A) R_{i^{*}}(s) Z(s), \\
F_{i, \beta_{i^{*}+1}}(s) & =-\alpha A R_{i^{*}}(s) Y(s), & G_{i, \beta_{i^{*}+1}}(s) & =-\alpha A R_{i^{*}}(s) Z(s),
\end{array}
$$

where

$$
\begin{aligned}
W(s) & =\frac{B(s)}{\operatorname{det} M(s)}-\frac{B(-s)}{\operatorname{det} M(-s)}=\frac{2 c\left[b_{2} c^{2} s^{2}+b_{2}-b_{0}\right]}{|\operatorname{det} M(s)|^{2}}, \\
X(s) & =\frac{B(s)}{\operatorname{det} M(s)}+\frac{B(-s)}{\operatorname{det} M(-s)}=\frac{2\left[\left(b_{2}+b_{1}\right) c^{2} s^{2}+b_{2}+b_{1}+b_{0}\right]}{|\operatorname{det} M(s)|^{2}}, \\
Y(s) & =\frac{1}{\operatorname{det} M(s)}-\frac{1}{\operatorname{det} M(-s)}=\frac{2 c\left[2 b_{2}+b_{1}\right]}{|\operatorname{det} M(s)|^{2}}, \\
Z(s) & =\frac{1}{\operatorname{det} M(s)}+\frac{1}{\operatorname{det} M(-s)}=\frac{2\left[-b_{2} c^{2} s^{2}+b_{2}+b_{1}+b_{0}\right]}{|\operatorname{det} M(s)|^{2}},
\end{aligned}
$$

and

$$
\begin{array}{rlrl}
Q & =k \alpha^{2} A(1-A), \quad \operatorname{det} M(s) & =b_{2}(s) B(s)^{2}+b_{1}(s) B(s)+b_{0}(s)=a(s)+i b(s), \\
R_{i}(s) & =2 k D_{i} C(s), & a(s) & =\left(1-c^{2} s^{2}\right) b_{2}(s)+b_{1}(s)+b_{0}(s), \\
k & =\frac{1}{1-\alpha^{2}}, & b(s) & =-c s\left(2 b_{2}(s)+b_{1}(s)\right), \\
B(s) & =1-i c s, & b_{0}(s) & =4 k D_{1} D_{2} C^{2}(s), \\
C(s) & =\cos (s)-1, & b_{1}(s) & =-2 k C(s)\left(D_{1}+D_{2}\right), \\
E(s) & =1-e^{i s}, & b_{2}(s) & =1-2 k \alpha^{2} A(1-A) C(s) .
\end{array}
$$

Notice that the set $M_{i}$ consists of all values at which the first derivative of $\varphi_{i}$ is discontinuous. This agrees with our prediction based on inspection of the equations (2.7).

For convenience, we will suppress the $s$-dependence of the functions $a, b, b_{0}, b_{1}$, and $b_{2}$. Our next proposition establishes some basic properties of the $\varphi_{i}$, but to do this, we need to bound the integrands in the solutions (2.8). This is accomplished by the following lemma.

Lemma 2.1. Let $\alpha \in[0,1), A \in[0,1), \beta \in \mathbb{R}$, and $c \neq 0$. Then

$$
|\operatorname{det} M(s)|^{2} \geq 1
$$

for $s \in \mathbb{R}$.

Proof. We leave the proof of this lemma to the second appendix.

Proposition 2.2. Use the definition of $\varphi_{i}$ as given in (2.8), and let $A \in$ $[0,1), \alpha \in[0,1), \beta \in \mathbb{R}, \xi \in \mathbb{R}$, and $c \neq 0$. Then $\varphi_{i}$ is continuous in $A, \alpha, \beta, \xi$, and $c$.

Proof. The previous lemma gives us a lower bound on the denominator of the integrands in (2.8), except when $s=0$. However, the $s$ appears in the denominator 
only when there is a sine in the numerator. The numerators of the integrands are also bounded, and from the definitions (2.8), we see that the integrands are continuous in the variables listed above. Also note from the definitions in (2.10) and (2.11) that the integrands are $O\left(s^{-2}\right)$ as $s \rightarrow \infty$, making the integral in (2.8) absolutely convergent. The continuity claims follow from this fact.

\section{Existence.}

3.1. Proof of existence. Recall that the candidate solutions were derived using the assumptions given in (2.6).

TheOREm 3.1. Let $A \in[0,1), \beta \in \mathbb{R}$, and $c \neq 0$, and consider our solutions as functions of $\alpha$ and $\xi$. That is, let $\varphi_{i}=\varphi_{i}(\alpha, \xi)$. Also recall $\beta_{1}=0$ and $\beta_{2}=\beta$. Suppose the following conditions are met:

C1. The $\varphi_{i}$ are continuous in $\alpha$ for all $\xi \in \mathbb{R}$ and for $\alpha \in[0,1)$.

C2. There exists $\delta=\delta\left(A, \beta, D_{1}, D_{2}, c\right)>0$ such that $\varphi_{i}^{\prime}(0, \xi)>0$ for $\xi \neq \beta_{i}$ in the interval $\left(\beta_{i}-\delta, \beta_{i}+\delta\right)$ (where' indicates differentiation with respect to $\xi$ ).

Then there exists a range of $\alpha$ for which $\varphi_{i}(\alpha, \xi)>a_{i}$ when $\xi>\beta_{i}$ and $\varphi_{i}(\alpha, \xi)<$ $a_{i}$ when $\xi<\beta_{i}$,

Proof. Consider the functions

$$
h_{i}(\alpha, \xi)=\varphi_{i}(\alpha, \xi)-\varphi_{i}\left(\alpha, \beta_{i}\right) .
$$

From the work done in [6], we know that $h_{i}(0, \xi)>0$ for $\xi>\beta_{i}$ and $h_{i}(0, \xi)<0$ for $\xi<\beta_{i}$ for $i=1,2$. We also know that the $h_{i}$ are continuous in $\alpha$ since, by Proposition 2.2, the $\varphi_{i}$ are continuous in $\alpha$. These two facts, together with the boundary conditions, guarantee that there is an $\alpha^{*}$ such that for $\alpha \in\left[0, \alpha^{*}\right), h_{i}(\alpha, \xi)>$ 0 for $\xi \geq \beta_{i}+\delta$ and $h_{i}(\alpha, \xi)<0$ for $\xi \leq \beta_{i}-\delta$. So let $\alpha \in\left[0, \alpha^{*}\right)$. To ensure that $h_{i}(\alpha, \xi)>0$ for $\xi \in\left(\beta_{i}, \beta_{i}+\delta\right)$ and $h_{i}(\alpha, \xi)<0$ for $\xi \in\left(\beta_{i}-\delta, \beta_{i}\right)$, we must also require that $\varphi_{i}^{\prime}(0, \xi)>0$ for $\xi \in\left(\beta_{i}-\delta, \beta_{i}+\delta\right) \backslash\left\{\beta_{i}\right\}$ (where, once again, ' denotes differentiation with respect to $\xi)$. So, condition $\mathrm{C} 2$ gives us that $\varphi_{i}(0, \xi)>0$ for $\xi \in\left(\beta_{i}-\delta, \beta_{i}+\delta\right) \backslash\left\{\beta_{i}\right\}$, which satisfies the requirement given above.

It remains to verify the hypotheses of Theorem 3.1. The strict monotonicity result from the $\alpha=0$ case done in [6] is enough to satisfy condition C2 (this is true because when $\alpha=0$, the solution is the same, up to a translation, for all $\beta$ ) and Proposition 2.2 satisfies condition $\mathrm{C} 1$. We now verify that the $\varphi_{i}$ satisfy our boundary conditions.

Proposition 3.2. For $i=1,2$,

$$
\lim _{\xi \rightarrow-\infty} \varphi_{i}(\xi)=0 \quad \text { and } \quad \lim _{\xi \rightarrow+\infty} \varphi_{i}(\xi)=1 .
$$

Proof. Recall the definition of $\varphi_{i}$ given in (2.8), and assume $\xi>0$. Now use the change of variables $s \rightarrow \frac{s}{\xi}$ and take the limit as $\xi \rightarrow+\infty$, using the Lebesgue dominated convergence theorem. We have

$$
\begin{aligned}
\lim _{\xi \rightarrow+\infty} \varphi_{i}(\xi)= & \frac{1}{2}+\frac{1}{2 \pi} \lim _{\xi \rightarrow+\infty} \int_{0}^{\infty} \sum_{j \in M_{i}}\left[\frac{1}{\xi} F_{i, j}\left(\frac{s}{\xi}\right) \cos \left(s-\frac{s j}{\xi}\right)\right. \\
& \left.+G_{i, j}\left(\frac{s}{\xi}\right) \frac{1}{s} \sin \left(s-\frac{s j}{\xi}\right)\right] d s \\
= & \frac{1}{2}+\frac{1}{2 \pi} \int_{0}^{\infty} \sum_{j \in M_{i}}\left[0 \cdot F_{i, j}(0) \cos (s)+G_{i, j}(0) \frac{1}{s} \sin (s)\right] d s \\
= & \frac{1}{2}+\frac{1}{2 \pi} \int_{0}^{\infty} \frac{\sin (s)}{s} X(0) d s=\frac{1}{2}+\frac{1}{\pi} \int_{0}^{\infty} \frac{\sin (s)}{s} d s=1 .
\end{aligned}
$$


Proof for the limit as $\xi \rightarrow-\infty$ is the same, except we assume $\xi<0$. But note that when $\xi<0$, the change of variables $s \rightarrow \frac{s}{\xi}$ results in the limits of integration becoming zero to $-\infty$, and everything else remains unchanged. The last expression then becomes

$$
\frac{1}{2}+\frac{1}{\pi} \int_{0}^{-\infty} \frac{\sin (s)}{s} d s=\frac{1}{2}-\frac{1}{\pi} \int_{-\infty}^{0} \frac{\sin (s)}{s}=0,
$$

giving us the result.

To summarize, we know there is an $\alpha^{*}>0$ such that solutions exist for all $\alpha \in$ $\left[0, \alpha^{*}\right)$. This next proposition gives a condition that implies our candidate solutions are not solutions of $(2.7)$.

Proposition 3.3. Fix $A \in[0,1), \alpha \in[0,1), \beta \in \mathbb{R}$, and $c \neq 0$. If either $\varphi_{i}\left(\beta_{i}\right)>1$ or $\varphi_{i}\left(\beta_{i}\right)<0$, then the candidate solutions given in (2.8) are not solutions of $(2.7)$.

Proof. By Proposition 3.2, $\varphi_{i}(\xi) \rightarrow 1$ as $\xi \rightarrow \infty$. Hence if $\varphi_{i}\left(\beta_{i}\right)>1$, there will be a $\xi>\beta_{i}$ for which $\varphi_{i}(\xi)<\varphi_{i}\left(\beta_{i}\right)$, violating our original assumptions (2.6). Recall also that $\varphi_{i}(\xi) \rightarrow 0$ as $\xi \rightarrow-\infty$, so if $\varphi_{i}\left(\beta_{i}\right)<0$, we have violated the assumption that $\varphi_{i}(\xi)<\varphi_{i}\left(\beta_{i}\right)$ for all $\xi<\beta_{i}$.

Having proved the existence of our solutions, we turn our attention to uncovering some basic properties of these solutions and to plots of several solution curves.

3.2. Plots of waveforms. We wish to show the form of the traveling wave solutions, but first, a few comments about our numerical studies are in order. Because we have an explicit formula for our candidate solutions, we are able to compute a broad range of numerical results. That said, we must also note that the large number of parameters in this problem makes it unfeasible to completely canvass the parameter space. Instead, we have focused on parameter values we expect will be representative of a larger range of values or on those that illustrate interesting phenomena. We approximate the integrals using the adaptive Gaussian quadrature code adapt of [19] and to find zeros we use the combined secant/bisection code zero of [19].

In Theorem 3.1, we proved the existence of solutions for some range of $\alpha$. The size of this range is unknown, and it is important to keep in mind that the existence of our candidate solutions may not be guaranteed for all combinations of parameter values explored in this section. We have, however, checked the necessary condition that $a_{i} \in(0,1)$, as mentioned in Proposition 3.3 for most of the parameter values in this section. In some instances, we have taken the additional step of numerically verifying (an admittedly finite range of) the candidate solutions themselves to verify that they satisfy our original assumptions. In particular, we have numerically determined waveforms with $D_{1}=D_{2}=1, A=\beta=0$, and $c$ values of $10^{-1}, 10^{0}$, and $10^{1}$, for $\alpha$ between 0 and .95 , with a step size of .05 .

Our intent in providing numerical results is twofold: to illustrate some of the results from the theoretical sections and to use numerical evidence to extend our knowledge of the problem and to possibly provide ideas for further study. (In particular, we wish to answer, at least tentatively, the questions of the effects of ephaptic coupling and nonalignment on the propagation of action potentials.) Figure 2 shows plots of wave forms for a small wave speed. Notice the large jumps; these correspond to discontinuities in the first derivative of the functions $\varphi_{i}$. A larger wave speed results in waves with much less pronounced jumps. An example of wave forms with larger $c$ is given later in Figure 6, but we withhold these plots for now since we will use them to illustrate a different phenomenon. 

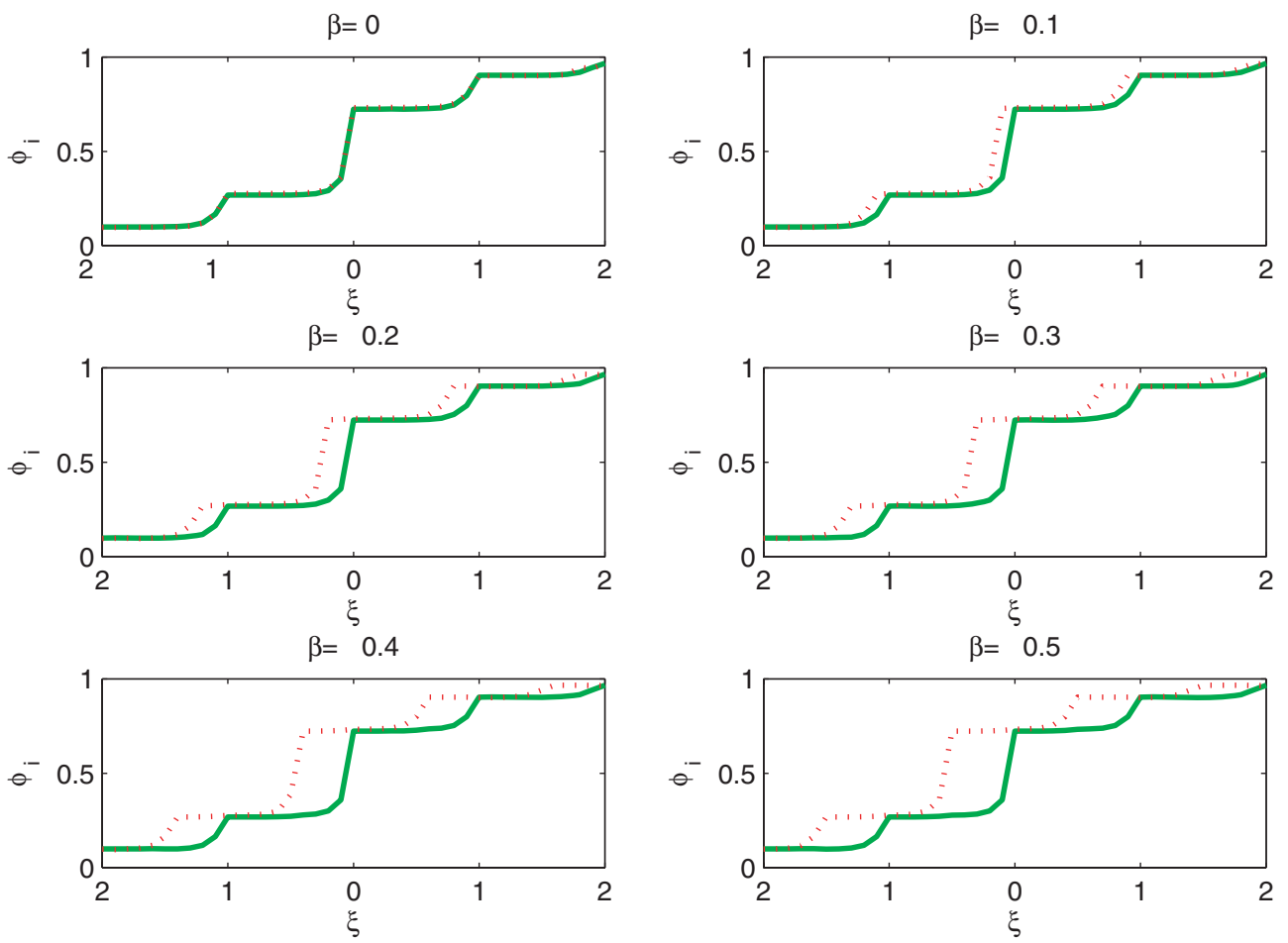

FIG. 2. Wave profiles for $c=.1, A=.5, \alpha=.1$, and $D_{1}=D_{2}=1$. The steep jumps are due to the small wave speed.

4. Some properties of the solution. Recall that, from the outset, we have required both $\varphi_{1}$ and $\varphi_{2}$ to have the same wave speed $c$, because we are interested in solutions that move together. Also recall that we are declaring $c$ and then allowing this choice of $c$ to determine the values for $a_{i}$. The idea is this: given a wave speed $c$, we want to know how much the detuning parameters will have to change under different circumstances in order for the waves to stay together. We start by writing the $a_{i}$ as functions of $c$ and then by investigating the behavior of these functions. In particular, to find the range of propagation failure, we will compute the value of $a_{i}$ as $c$ approaches zero; this will tell us which values of $a_{i}$ can support a nonzero wave speed. We also give an expression for the rate of change in the range of propagation failure when $\alpha$ moves away from zero, and we evaluate this expression explicitly in a few special cases.

4.1. Plots of $\boldsymbol{a}_{\boldsymbol{i}}(\boldsymbol{c})$ curves. Recall that from our original assumptions (2.6), we have that

$$
a_{i}(c)=\varphi_{i}\left(\beta_{i}\right) .
$$

In Figures 3 and 4 we present $a_{i}(c)$ curves, which relate a given wave speed to the detuning parameters $a_{i}$. Take special note of the distance between $a_{i}(c)$ and $\frac{1}{2}$ at $c=0$ : this is the range of propagation failure mentioned several times already. Also note that smaller values of the diffusion constants $D_{i}$ result in larger ranges of propagation failure. For $D_{1}=D_{2}$, increasing the value of the $D_{i}$ results in a smaller value of the detuning parameter required to achieve a certain wave speed. 

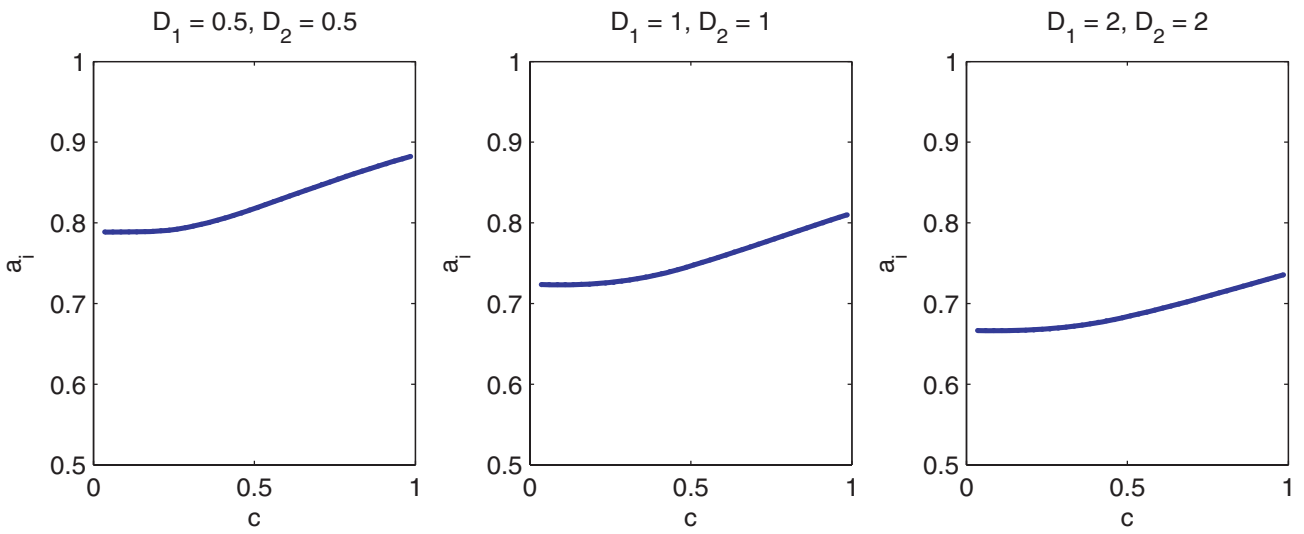

FIG. 3. $a_{i}(c)$ curves for $A=.5, \beta=-.5$, and $\alpha=0.1$. In this case, $a_{1}=a_{2}$. Notice that $\lim _{c \rightarrow 0} a_{i}(c)$ is significantly greater than $\frac{1}{2}$, implying a nontrivial range of propagation failure. Also notice that, for a given $c, a_{i}(c)$ decreases as $D_{1}=D_{2}$ increases. Similar plots result in the case $A=0=\beta$.
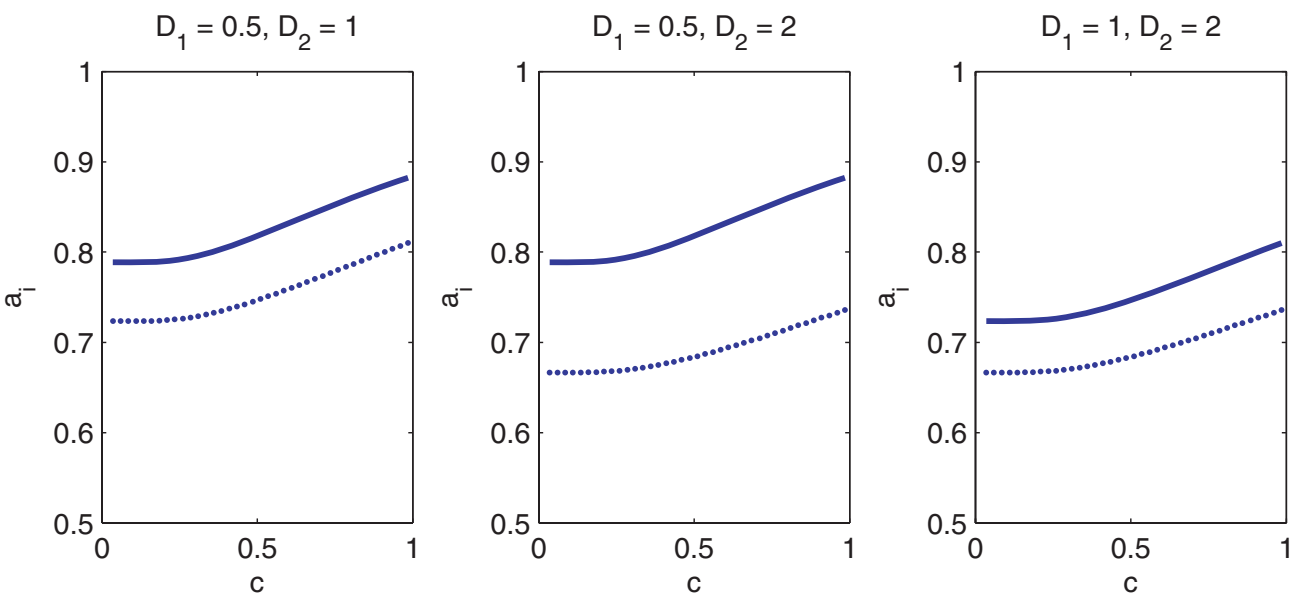

FIG. 4. $a_{i}(c)$ curves for $A=.5, \beta=-.5$, and $\alpha=0.1$, when $D_{1} \neq D_{2}$. The solid line represents $a_{1}$, and the dotted line represents $a_{2}$. Similar plots result in the case $A=0=\beta$.

4.2. Analytical results related to the $\boldsymbol{a}_{i}(c)$ relationship. We comment on notation: some of the functions introduced in this section depend on several parameters; since there are so many parameters, we consider all parameters to be fixed except the ones listed explicitly in the argument of the function. Now consider the quantity

$$
\Gamma_{i}(c)=a_{i}(c)-\frac{1}{2}=\frac{1}{2 \pi} \int_{0}^{\infty} \sum_{j \in M_{i}} F_{i, j}(s) \cos \left(s\left(\beta_{i}-j\right)\right)+G_{i, j}(s) \frac{1}{s} \sin \left(s\left(\beta_{i}-j\right)\right) d s .
$$

Also define

$$
g(c)=a_{1}(c)-a_{2}(c)=\Gamma_{1}(c)-\Gamma_{2}(c),
$$

and note that this quantity represents the difference in the detuning parameters $a_{i}$ required to make both waves travel at speed $c$. This definition allows us to state several important questions: 1 . Given a noncoupled system with certain parameter 
values, what happens to the quantity $g$ when the coupling coefficient $\alpha$ moves away from zero? 2. Given a coupled, aligned system with certain parameter values, what happens to the quantity $g$ when the alignment coefficient $A$ moves away from zero? In this section we begin to answer these questions.

The following results about the oddness of the $\Gamma_{i}$ illustrate the symmetry held by these functions under certain circumstances.

Proposition 4.1. Let $\Gamma_{i}(c)$ be defined as in (4.2), and let $c \neq 0$. Then $\Gamma_{i}$ is odd in $c$ if and only if

$$
\int_{0}^{\infty} \sum_{j \in M_{i}} G_{i, j}(s) \frac{1}{s} \sin \left(s\left(\beta_{i}-j\right)\right) d s=0
$$

for all $c \neq 0$. Further,

$$
\lim _{c \rightarrow \pm \infty} \Gamma_{i}(c)=\frac{1}{2}
$$

Proof. Note that $X(s)$ and $Z(s)$, as given in (2.10), are even with respect to $c$, $W(s)$ and $Y(s)$ are odd with respect to $c$, and all other terms in $\Gamma_{i}$ are independent of $c$. If the condition (4.4) holds, then we see from the definitions of $F_{i, j}$ and $G_{i, j}$ that all appearances of $X(s)$ and $Z(s)$ vanish, leaving us with $\Gamma_{i}$ odd. Conversely, if the condition does not hold, then the dependence of $\Gamma_{i}$ on $c$ is not odd.

For the second statement, we use the change of variables $s \rightarrow \frac{s}{c}$, interchange the limiting process with the integration, and then evaluate the result:

$$
\begin{aligned}
\lim _{c \rightarrow+\infty} \Gamma_{i}(c) & =\frac{1}{2 \pi} \int_{0}^{\infty} \lim _{c \rightarrow \infty} \sum_{j \in M_{i}} \cos \left(\frac{-s j}{c}\right) F_{i, j}\left(\frac{s}{c}\right) d s \\
& =\frac{1}{2 \pi} \int_{0}^{\infty} \lim _{c \rightarrow \infty} W\left(\frac{s}{c}\right) d s \\
& =\frac{1}{\pi} \int_{0}^{\infty} \frac{s^{2}+1}{s^{4}+2 s^{2}+1} d s=\frac{1}{2} .
\end{aligned}
$$

Once again, the limit as $c \rightarrow-\infty$ is evaluated in the same way, but the limits of integration change, much like in the proof of Proposition 3.2, giving us the result stated above.

The second claim in this last theorem implies that increasing the wave speed to arbitrarily large values requires the detuning parameters to be very close to zero or one.

We also have the following corollary.

COROLlary 4.2. In particular, $\Gamma_{i}$ is odd in the following cases:

1. $\alpha=0$,

2. $A=0$ and $\beta=0$,

3. $A=\frac{1}{2}$ and $\beta=-\frac{1}{2}$.

Proof. Conditions 1, 2, and 3 all imply (4.4) for all $c \neq 0$.

Note that conditions 1,2 , and 3 are enough to imply the oddness of $\Gamma_{i}$, regardless of the values of the other parameters. It is not clear, however, whether there exist other solutions to the equation in (4.4), much less whether there exist other solutions that are independent of the other parameters. 
Now consider the functions

$$
\begin{aligned}
P_{Y}(c) & =\frac{1}{2 \pi} \int_{0}^{\infty} \sum_{j \in M_{1}} F_{1, j}(s) \cos \left(s\left(\beta_{1}-j\right)\right)-\sum_{j \in M_{2}} F_{2, j}(s) \cos \left(s\left(\beta_{2}-j\right)\right) d s \\
& =\frac{k}{\pi} \int_{0}^{\infty} Y(s) C(s)\left[D_{1}-D_{2}\right][1+\alpha(1-A) \cos (s \beta)+\alpha A \cos (s(\beta+1))] d s, \\
P_{Z}^{*}(c) & =\frac{1}{2 \pi} \int_{0}^{\infty} \sum_{j \in M_{1}} G_{1, j}(s) \sin \left(s\left(\beta_{1}-j\right)\right)-\sum_{j \in M_{2}} G_{2, j}(s) \sin \left(s\left(\beta_{2}-j\right)\right) d s \\
& =\frac{k}{\pi} \int_{0}^{\infty} \frac{1}{s} Z(s) C(s)\left[D_{1}+D_{2}\right][(1-A) \sin (s \beta)+A \sin (s(\beta+1))] d s .
\end{aligned}
$$

In fact an $\alpha$ factors out of $P_{Z}^{*}$, so we will denote

$$
P_{Z}(c)=\frac{P_{Z}^{*}(c)}{\alpha}
$$

which allows us to write

$$
g(c)=P_{Y}(c)+\alpha P_{Z}(c),
$$

where $g(c)$ is the difference between the detuning parameters $a_{1}$ and $a_{2}$, as given in (4.3). This representation of $g$ will be helpful in proving the following results.

TheOREM 4.3. Let $g$ be defined as above. If either $\alpha=0$ or $P_{Z}(c)=0$, then

$$
c>0 \Rightarrow g(c)\left\{\begin{array}{lll}
<0 & \text { for } & D_{1}>D_{2}, \\
=0 & \text { for } & D_{1}=D_{2}, \\
>0 & \text { for } & D_{1}<D_{2},
\end{array} \quad c<0 \Rightarrow g(c)\left\{\begin{array}{lll}
>0 & \text { for } & D_{1}>D_{2}, \\
=0 & \text { for } & D_{1}=D_{2}, \\
<0 & \text { for } & D_{1}<D_{2} .
\end{array}\right.\right.
$$

Proof. Clearly, if either assumption is true, then $g(c)=P_{y}(c)$. The only remaining task is to determine the sign of $P_{y}(c)$. To do this, note that $Y(s, c)>0$ when $c>0$ and $Y(s, c)<0$ when $c<0$ and that $C(s) \leq 0$. Further, we have that

$$
\begin{array}{r}
1+\alpha(1-A) \cos (s \beta)+\alpha A \cos (s(\beta+1)) \\
>1-\alpha(1-A)-\alpha A=1-\alpha>0,
\end{array}
$$

and these estimates give the result above.

The previous theorem determines the sign of $g$ for a number of cases, since if either condition 2 or condition 3 from Corollary 4.2 is met, then $P_{Z}(c) \equiv 0$. The following results provide more examples of the symmetries of the functions $\Gamma_{i}$ and $g$.

Theorem 4.4. Fix $\alpha \in[0,1)$. Then for $A \in(0,1)$,

$$
\Gamma_{i}\left(D_{1}, D_{2}, c, A, \beta\right)=-\Gamma_{i^{*}}\left(D_{2}, D_{1},-c, A, \beta\right)=\Gamma_{i^{*}}\left(D_{2}, D_{1}, c, 1-A,-\beta-1\right),
$$

and for $A=0$,

$$
\Gamma_{i}\left(D_{1}, D_{2}, c, 0, \beta\right)=-\Gamma_{i^{*}}\left(D_{2}, D_{1},-c, 0, \beta\right)=\Gamma_{i^{*}}\left(D_{2}, D_{1}, c, 0,-\beta\right) .
$$

Proof. Follows from the definitions of the functions $F_{i, j}$ and $G_{i, j}$ given in (2.9) and the definition of $\Gamma_{i}$ given in (4.2).

Corollary 4.5. Fix $\alpha \in[0,1)$. Then for $A \in(0,1)$,

$$
\Gamma_{i}\left(D_{1}, D_{2}, c, A, \beta\right)=-\Gamma_{i}\left(D_{1}, D_{2},-c, 1-A,-\beta-1\right) \quad \text { and }
$$



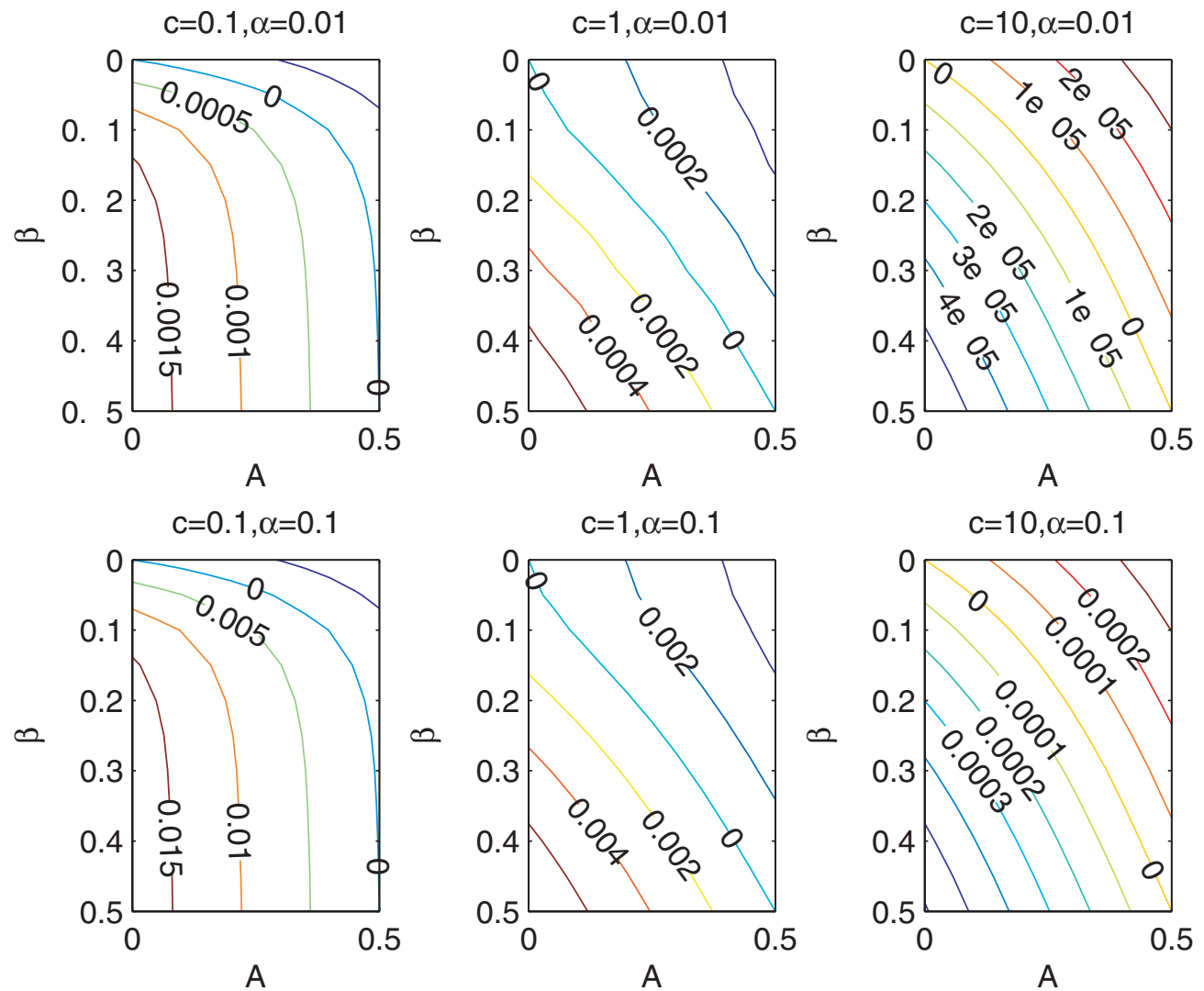

FIG. 5. Contours of $g(A, \beta)$ for $D_{1}=D_{2}=1$. Notice that for all $A \in\left[0, \frac{1}{2}\right]$, there is a $\beta$ such that $g(A, \beta)=0$.

$$
\begin{aligned}
g\left(D_{1}, D_{2}, c, A, \beta\right) & =-g\left(D_{2}, D_{1}, c, 1-A,-\beta-1\right) \\
& =-g\left(D_{1}, D_{2},-c, 1-A,-\beta-1\right)=g\left(D_{2}, D_{1},-c, A, \beta\right),
\end{aligned}
$$

and for $A=0$,

$$
\begin{gathered}
\Gamma_{i}\left(D_{1}, D_{2}, c, 0, \beta\right)=-\Gamma_{i}\left(D_{1}, D_{2},-c, 0,-\beta\right) \quad \text { and } \\
g\left(D_{1}, D_{2}, c, 0, \beta\right)=-g\left(D_{2}, D_{1}, c, 0,-\beta\right) \\
=-g\left(D_{1}, D_{2},-c, 0,-\beta\right)=g\left(D_{2}, D_{1},-c, 0, \beta\right) .
\end{gathered}
$$

Proof. The proof follows from Theorem 4.4 and the definitions of $\Gamma_{i}$ and $g$ given in (4.2) and (4.3).

Note that (4.14), (4.16), and (4.17) are also true for $A=0$, but the statements given in (4.15), (4.19), and (4.20) are better results. We turn our attention now to the issue of nonalignment.

4.3. Numerical results related to nonalignment. We mentioned earlier that it is important to note which values of the parameters cause $a_{1}=a_{2}$. This is equivalent, of course, to finding the zeros of our function $g$. The contour plots in Figure 5 

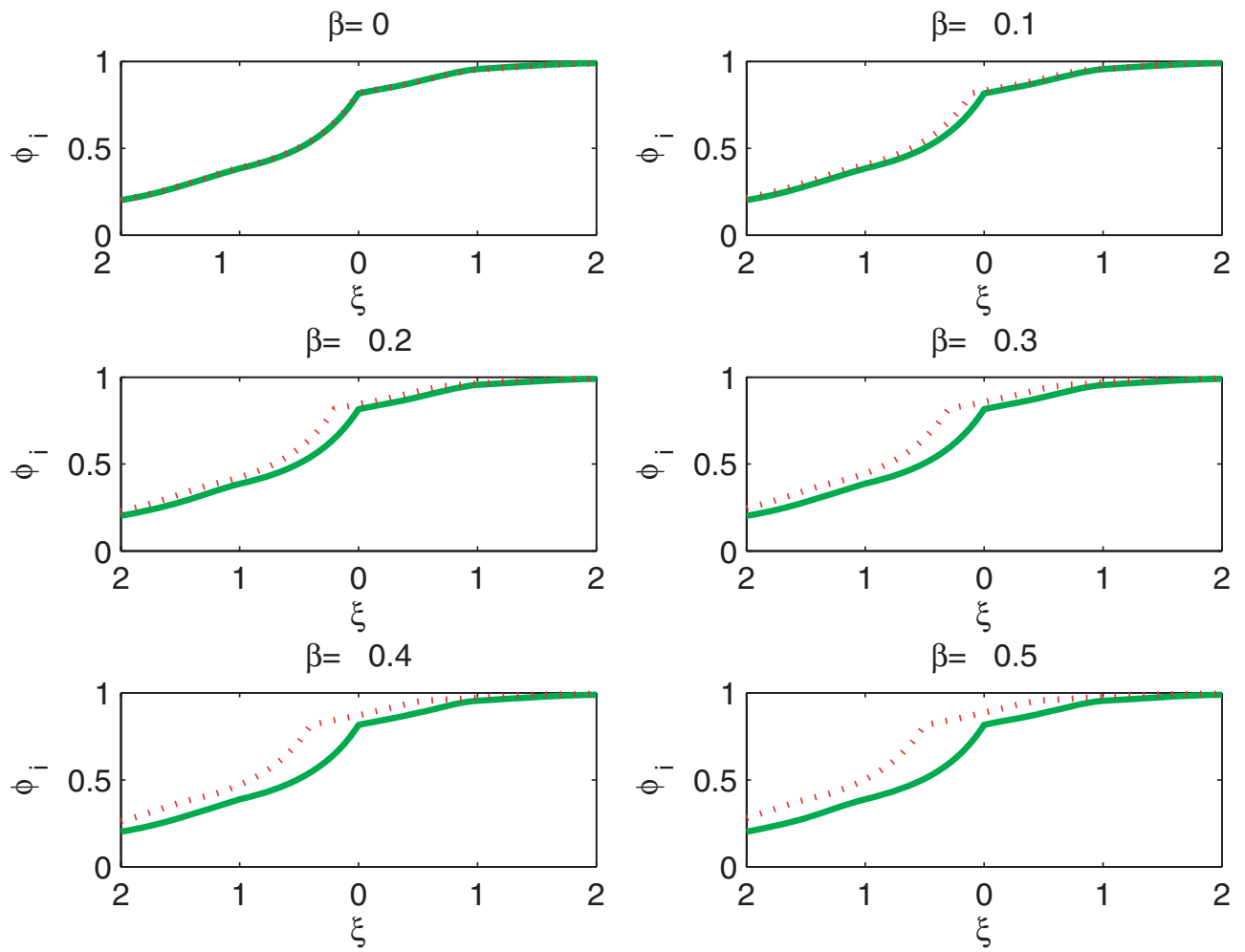

FIG. 6. Wave profiles for $D_{1}=D_{2}=1, \alpha=.1, c=1$, and $A=.3$. The cusps in the graphs at $a_{i}\left(\beta_{i}\right)$ are the result of the Heaviside functions switching from 0 to 1 . The solid line represents $\varphi_{1}$, and the dotted line represents $\varphi_{2}$.

of $g=0$ show that, given conditions with $A=0$ that yield $g=0$, we may move $A$ away from zero, and, as long as we adjust $\beta$ appropriately, $g$ will remain zero.

For example, consider the bottom middle plot, in which $c=1$ and $\alpha=.1$. When $A=0=\beta$, we see that $g(A, \beta)=0$. In addition, for any $A \in\left[0, \frac{1}{2}\right]$, there is a $\beta \in\left[-\frac{1}{2}, 0\right]$ such that $g(A, \beta)=0$. we see that this last statement is true of all plots in Figure 5 , although we have not proved that such a $\beta$ exists in $\left[-\frac{1}{2}, 0\right]$ in all circumstances.

Here we will fix $\alpha$ and examine the effects of moving the alignment parameter $A$ away from zero. Recall our earlier mentioning of the parameter $\beta$ as a time-delay. It is our contention that, loosely speaking, problems caused by moving $A$ away from zero can be remedied by moving $\beta$ a corresponding (but not necessarily equal) distance from zero. Consider, for instance, the case $\beta=0$. Recalling our traveling wave ansatz $\xi=n-c t$, we see that at time $t=0$, for instance, $\xi=0$ will correspond with $n=0$ for both fibers. However, if $A>0$, then wave 2 will be "ahead" of wave 1 . To make this notion a little more precise, we will speak of the "location" of a wave as being at $\beta_{i}$. Moving $\beta$ below zero corrects this problem by having wave 2 reach a given node at a time $\beta$ later than wave 1 reaches the same node. The upper left plot in Figure 6 gives an example of two waves that are lined up with respect to $\xi$ when $\beta=0$. Since $A \neq 0$, however, this lining up actually corresponds to one wave leading the other. It is only when $\beta$ is moved below zero that the waves travel together, as indicated by the other plots in Figure 6. 

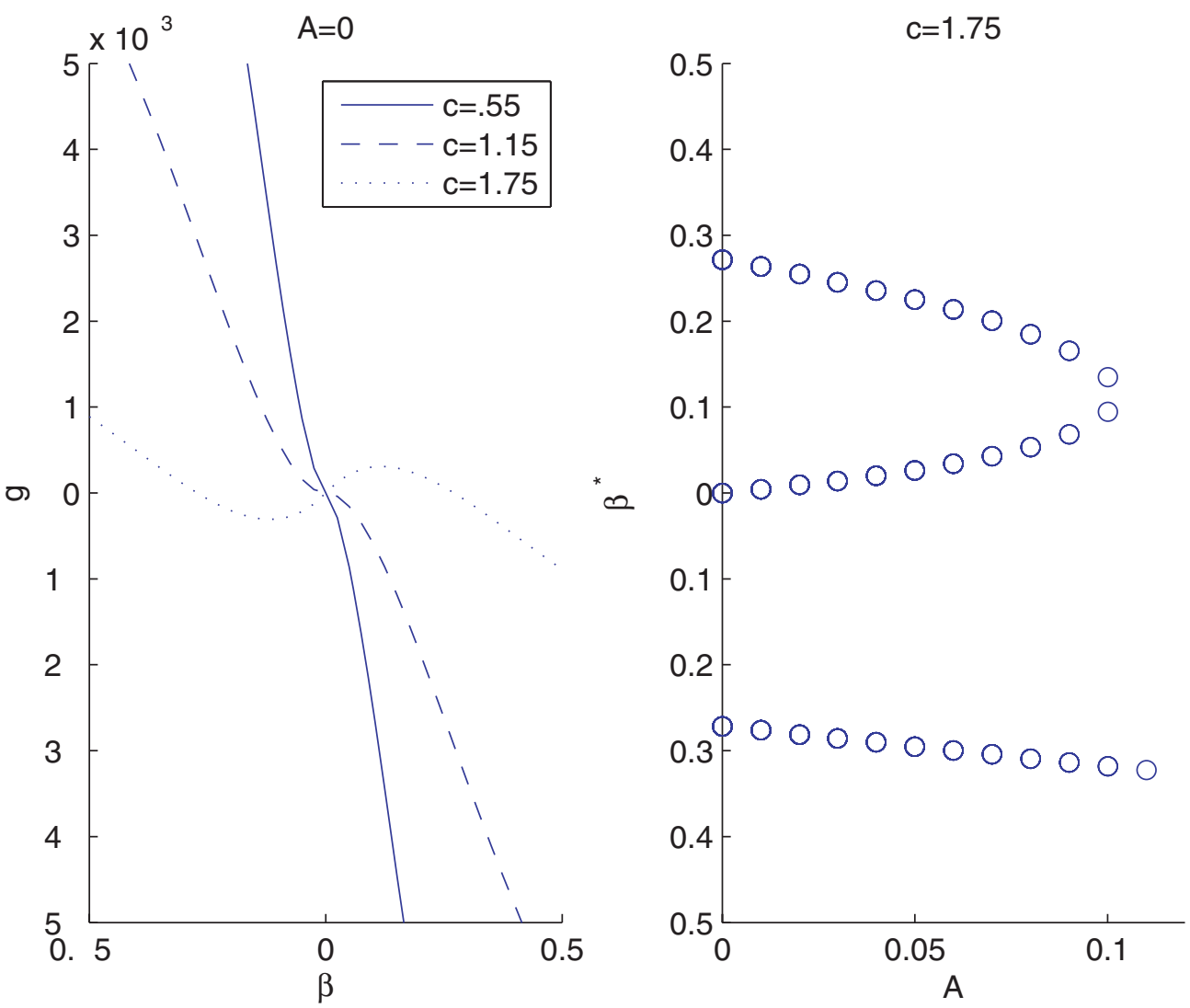

FIG. 7. Left: plots of $g(\beta)$ for $A=0$. When $c=1.75$, we see that $g(\beta)=0$ for three different values of $\beta$. Right: zeros of $g(\beta)$ for $c=1.75$. This plot shows how the three zeros shown in the left plot for $c=1.75$ change as $A$ changes. In both plots, $D_{1}=D_{2}=1$ and $\alpha=.1$.

The relationship between $A$ and $\beta$ has been relatively unexplored analytically. We want to know if $A$ and $\beta$ are independent of each other, or if fixing a value of $A$ determines a value of $\beta$. Consider an uncoupled system in which $D_{1}=D_{2}$, and note that this is equivalent to the condition $a_{1}=a_{2}$. We want to know, given fixed values of $A$ and $\alpha>0$, whether there exists a critical value of $\beta$ that still results in $a_{1}=a_{2}$. This section provides evidence that such a $\beta$ does exist for a variety of combinations of $A, \alpha$ and $c$ (see Tables 4.1 and 4.2).

Further, these numerical results show that multiple such $\beta$ values exist for certain combinations of these parameters, particularly for higher wave speeds. We find evidence for the existence of a pitchfork bifurcation in the parameter $c$ with respect to these critical $\beta$ values. That is, fixing all parameters but $c$, there exists a $c^{*}$ (dependent on the other parameters) such that for $c \leq c^{*}$ there is only one critical $\beta$ value, but that for $c>c^{*}$, there are three critical $\beta$ values. The left plot in Figure 7 shows the cubic-like shape of $g(\beta)$ and shows the transition between $g(\beta)$ having one zero to $g(\beta)$ having three zeros in the range shown. The plot on the right shows implicitly the dependence of this value $c^{*}$ on the parameter $A$ in particular. For smaller values of $A$, a wave speed of $c=1.75$ results in three critical $\beta$ values, whereas for large enough values of $A$, there is only one critical $\beta$ value for which $g(\beta, c)=0$. The possible implications of a pitchfork bifurcation are interesting. For instance, if the 
TABLE 4.1

Values of $\beta$ that yield $a_{1}=a_{2}$ for $\alpha=.1, D_{1}=D_{2}=1$, and different pairs of the wave speed $c$ and the alignment parameter $A$.

\begin{tabular}{|c|c|c|c|c|c|c|}
\hline $\mathrm{c} \backslash \mathrm{A}$ & 0 & .1 & .2 & .3 & .4 & .5 \\
\hline .25 & 0 & -.0433 & -.0792 & -.1328 & -.2381 & -.5 \\
\hline .75 & 0 & -.0938 & -.1651 & -.2506 & -.3623 & -.5 \\
\hline 1.25 & 0 & -.1409 & -.2200 & -.3035 & -.3974 & -.5 \\
\hline 1.75 & 0 & -.3183 & -.3634 & -.4085 & -.4540 & -.5 \\
\hline
\end{tabular}

TABLE 4.2

Values of $a_{1}=a_{2}$ that result from the $\beta$ value listed in Table 4.1 for different pairs of the wave speed $c$ and the alignment parameter $A$.

\begin{tabular}{|c|c|c|c|c|c|c|}
\hline $\mathrm{c} \backslash \mathrm{A}$ & 0 & .1 & .2 & .3 & .4 & .5 \\
\hline .25 & .7346 & .7328 & .7311 & .7294 & .7277 & .7267 \\
\hline .75 & .7891 & .7878 & .7864 & .7852 & .7844 & .7840 \\
\hline 1.25 & .8521 & .8510 & .8500 & .8492 & .8487 & .8485 \\
\hline 1.75 & .8975 & .8959 & .8955 & .8952 & .8950 & .8949 \\
\hline
\end{tabular}

single traveling wave before the bifurcation is stable, which is reasonable to expect for small values of the coupling parameter $\alpha$ (see [7]), then we might expect an exchange of stability at the bifurcation point.

The existence of multiple $\beta$ values that result in $g=0$ suggests that perhaps the two waves can travel at the same speed even if one wave is lagging behind the other. This allows for the possibility of two waves traveling together even if their respective nerve fibers were not activated at exactly the same time. Other numerical results indicate that the value of $c^{*}$ increases as $A$ increases. This suggests that in the case of staggered nodes, higher wave speeds are required in order to have multiple critical $\beta$ values. The implications of this would be very interesting if there is in fact an exchange of stability at the bifurcation point. If the middle branch becomes unstable after the bifurcation, then this would imply that the middle branch stays stable for a larger range of the wave speed when the nodes are staggered than when they are aligned.

\section{Propagation failure.}

5.1. Analytical results on propagation failure. As mentioned earlier, propagation failure is a well-documented phenomenon in lattice differential equations (see [6] and [11], among others), and it is certainly important to compute the range of $a_{i}$ that result in a zero wave speed. We address this issue here. This section involves a few lengthy calculations, so we highlight the most important results of this section with a few theorems and then proceed with the calculations that lead to the results. To determine the range of propagation failure, we need to compute $\lim _{c \rightarrow 0} \Gamma_{i}(c)$. In light of the symmetries of $\Gamma_{i}$ presented previously, we will focus on the computation of $\lim _{c \rightarrow 0+} \Gamma_{i}(c)$. Also, we will start by considering only the case where $\Gamma_{i}(c)$ is odd, and the consideration of the other case will follow. These steps are carried out in the following proposition. Then

Proposition 5.1. Let $\alpha \in[0,1), A \in[0,1), \beta \in \mathbb{R}$, and $\gamma_{i}=\lim _{c \rightarrow 0+} \Gamma_{i}(c)$.

$$
\begin{aligned}
\gamma_{i}=\lim _{c \rightarrow 0+} \Gamma_{i}(c)= & \sum_{j \in M_{i}} \frac{1}{2 \pi p} \int_{0}^{\infty} \int_{0}^{p} F_{i, j}^{*}\left(\frac{t}{c}, s\right) \cos \left(s\left(\beta_{i}-j\right)\right) d s d t \\
& +\int_{0}^{\infty}\left[G_{i, j}(s)\right]_{c=0} \frac{1}{s} \sin (s(\xi-j)) d s
\end{aligned}
$$


Proof. We will compute $\lim _{c \rightarrow 0} \int_{0}^{\infty} F_{i, j}(s) \cos \left(s\left(\beta_{i}-j\right)\right) d s$ individually for a given $(i, j)$ pair. First, let's assume $\beta_{i}-j$ is rational, and then define

$$
p_{i, j}:=p_{i, j}^{*} 2 \pi:=\operatorname{lcm}\left(2 \pi, \frac{2 \pi}{\beta_{i}-j}\right),
$$

the common period of the periodic functions that define $F_{i, j}$, and

$$
p=\operatorname{lcm}\left(p_{i, j}\right),
$$

the least common multiple over all $i$ and $j$ of the $p_{i, j}$. For notational purposes, we consider $W, X, Y$, and $Z$ as functions of two variables, e.g., $W(s)=W(u, v)$ : the first argument will go in place of all nonperiodic instances of $s$, and the second argument will go in place of all periodic instances of $s$. As an example, we would write the function $q(s)=a s^{4}+b s^{2}+\sin (s)$ as $q(u, v)=a u^{4}+b u^{2}+\sin (v)$.

For the $F_{i, j}$ and $G_{i, j}$ we will use the same convention. So, for instance,

$$
F_{1,0}(u, v)=\left[W(u, v)(1+2 Q)-R_{2}(v) Y(u, v)\right],
$$

and $F_{i, j}(s, s)=F_{i, j}(s)$. Then

$$
\begin{aligned}
\frac{1}{2 \pi} \int_{0}^{\infty} F_{i, j}(s) \cos \left(s\left(\beta_{i}-j\right)\right) d s & =\frac{1}{2 \pi} \sum_{n=0}^{\infty} \int_{n p}^{(n+1) p} F_{i, j}(s) \cos \left(s\left(\beta_{i}-j\right)\right) d s \\
& =\frac{1}{2 \pi} \sum_{n=0}^{\infty}\left(\int_{n p}^{(n+1) p} F_{i, j}(n p, s) \cos \left(s\left(\beta_{i}-j\right)\right) d s+E_{n}\right) \\
: & =\Phi+\frac{1}{2 \pi} \sum_{n=0}^{\infty} E_{n},
\end{aligned}
$$

where

$$
E_{n}=\int_{n p}^{(n+1) p} F_{i, j}(s) \cos \left(s\left(\beta_{i}-\xi\right)\right) d s-\int_{n p}^{(n+1) p} F_{i, j}(n p, s) \cos \left(s\left(\beta_{i}-j\right)\right) d s
$$

and

$$
\Phi=\frac{1}{2 \pi} \sum_{n=0}^{\infty} \int_{n p}^{(n+1) p} F_{i, j}(n p, s) \cos \left(s\left(\beta_{i}-j\right)\right) d s .
$$

We will show in an appendix that

$$
\sum_{n=0}^{\infty} E_{n} \rightarrow 0 \quad \text { as } \quad c \rightarrow 0,
$$

but for now, we will assume this is true and complete the calculation of $\Phi$. From the definitions of $W$ and $Y$ given in (2.10), notice that $c$ factors out of the $F_{i, j}$. This allows us to write

$$
F_{i, j}(s)=c F_{i, j}^{*}(s),
$$


and we will denote

$$
P_{i, j}(t)=\frac{1}{p} \int_{0}^{p} F_{i, j}^{*}\left(\frac{t}{c}, s\right) \cos \left(s\left(\beta_{i}-j\right)\right) d s
$$

Then

$$
\begin{aligned}
\Phi & =\frac{c}{2 \pi} \sum_{n=0}^{\infty} \int_{n p}^{(n+1) p} F_{i, j}^{*}(n p, s) \cos \left(s\left(\beta_{i}-j\right)\right) d s \\
& =\frac{p c}{2 \pi} \sum_{n=0}^{\infty} \frac{1}{p} \int_{0}^{p} F_{i, j}^{*}(n p, s) \cos \left(s\left(\beta_{i}-j\right)\right) d s \\
& =\frac{p c}{2 \pi} \sum_{n=0}^{\infty} P_{i, j}(n p c) .
\end{aligned}
$$

Viewing this final quantity as a Riemann sum, we arrive at

$$
\Phi=\frac{1}{2 \pi} \int_{0}^{\infty} P_{i, j}(t) d t=\frac{1}{2 \pi p} \int_{0}^{\infty} \int_{0}^{p} F_{i, j}^{*}\left(\frac{t}{c}, s\right) \cos \left(s\left(\beta_{i}-j\right)\right) d s d t .
$$

This brings us to the expression, for $\Gamma_{i}$ odd,

$$
\gamma_{i}=\lim _{c \rightarrow 0} \Gamma_{i}(c)=\sum_{j} \frac{1}{2 \pi p} \int_{0}^{\infty} \int_{0}^{p} F_{i, j}^{*}\left(\frac{t}{c}, s\right) \cos \left(s\left(\beta_{i}-j\right)\right) d s d t .
$$

If $\Gamma_{i}$ is not odd, then we need to consider the effect of the $G_{i, j}$. However, this is much easier to compute since

$$
\lim _{c \rightarrow 0} \int_{0}^{\infty} G_{i, j}(s) \frac{1}{s} \sin (s(\xi-j)) d s=\int_{0}^{\infty}\left[G_{i, j}(s)\right]_{c=0} \frac{1}{s} \sin (s(\xi-j)) d s,
$$

and so we have the general formula

$$
\begin{aligned}
\gamma_{i}=\lim _{c \rightarrow 0} \Gamma_{i}(c)= & \sum_{j} \frac{1}{2 \pi p} \int_{0}^{\infty} \int_{0}^{p} F_{i, j}^{*}\left(\frac{t}{c}, s\right) \cos \left(s\left(\beta_{i}-j\right)\right) d s d t \\
& +\int_{0}^{\infty}\left[G_{i, j}(s)\right]_{c=0} \frac{1}{s} \sin (s(\xi-j)) d s
\end{aligned}
$$

One of our goals here is to investigate the changes in this quantity with respect to a change in $\alpha$ and, in particular, the difference in the range of propagation failure between when $\alpha=0$ and when $\alpha>0$. To this end, we let $\gamma_{i}=\gamma_{i}(\alpha)$, calculate $\gamma_{i}^{\prime}(\alpha)$, and evaluate this expression at $\alpha=0$. In a few special cases, we have an explicit value for $\gamma_{i}^{\prime}(\alpha)$ in terms of $D_{1}$ and $D_{2}$. The following theorem gives this result.

ThEOREM 5.1. Let $D_{1}=D_{2}:=D$, and consider the function $\gamma_{i}=\gamma_{i}(\alpha)$.

1. If $A=0=\beta$, then

$$
\gamma_{i}^{\prime}(0)=\frac{D}{(4 D+1)^{\frac{3}{2}}}>0
$$

2. If $A=\frac{1}{2}$ and $\beta=-\frac{1}{2}$, then

$$
\gamma_{i}^{\prime}(0)=0
$$


Recall that $\gamma_{i}=\lim _{c \rightarrow 0+} a_{i}(c)-\frac{1}{2}$. If $\gamma_{i}>0$, then there is a positive range of propagation failure. The results above quantify how the size of this range changes as $\alpha$ moves away from zero. Before we proceed with the proof of this theorem, let us interpret the results in terms of the ephaptic coupling model. This theorem states that turning the coupling on results in an increase in the range of propagation failure when the nodes are aligned, but that turning the coupling on results in a decrease in the range of propagation failure when the nodes are staggered. Intuitively, it seems reasonable that staggering the nodes would result in a decrease in this range. The two-fiber problem with staggering seems very much like the one-fiber problem with twice as many nodes squeezed into the same space. A shorter internodal distance is reflected by an increase in the diffusion coefficient, which has the effect of decreasing the range of propagation failure. As the coupling increases, the fibers interact more, increasing the effect of the shorter internodal distance.

The result for the aligned case is more easily understood by thinking about the system (2.7). When $A=0=\beta$, we are left with

$$
\begin{aligned}
& -c \varphi_{1}^{\prime}(\xi)=\frac{1}{1-\alpha^{2}}\left[D_{1}\left(L \varphi_{1}\right)(\xi)-\alpha D_{2}\left(L \varphi_{2}\right)(\xi)\right]-\varphi_{1}(\xi)+h(\xi), \\
& -c \varphi_{2}^{\prime}(\xi)=\frac{1}{1-\alpha^{2}}\left[-\alpha D_{1}\left(L \varphi_{1}\right)(\xi)+D_{2}\left(L \varphi_{2}\right)(\xi)\right]-\varphi_{2}(\xi)+h(\xi),
\end{aligned}
$$

and when $D_{1}=D_{2}$, we expect to have $\varphi_{1}=\varphi_{2}$. In this situation, the system would become two copies of the equation

$$
\begin{aligned}
-c \varphi^{\prime}(\xi) & =\frac{1}{1-\alpha^{2}}[D(1-\alpha)(L \varphi)(\xi)]-\varphi(\xi)+h(\xi) \\
& =\frac{D}{1+\alpha}(L \varphi)(\xi)-\varphi(\xi)+h(\xi),
\end{aligned}
$$

which is just the one-fiber problem with diffusion coefficient $\frac{D}{1+\alpha}$. This makes it easy to see that increasing $\alpha$ decreases the diffusion coefficient in this related one-fiber problem, which has the effect of increasing the range of propagation failure. Using the value of $\gamma$ computed for the one-fiber problem in [6], and viewing $\gamma$ as a function of $\alpha$, we have

$$
\gamma(\alpha)=\frac{1}{2\left(1+\frac{4 D}{1+\alpha}\right)^{\frac{1}{2}}}
$$

and

$$
\gamma^{\prime}(\alpha)=\frac{D}{(1+\alpha)^{2}\left(1+\frac{4 D}{1+\alpha}\right)^{\frac{3}{2}}} .
$$

In particular,

$$
\gamma^{\prime}(0)=\frac{D}{(1+4 D)^{\frac{3}{2}}},
$$

which is in agreement with the result obtained in the theorem. This formula also sheds light on how the range of propagation failure might decrease for all values of $\alpha$. Unfortunately, such an argument cannot be made when $A \neq 0$. However, the reasoning used here works for any nonlinearity $f$ for which the wave speed increases 
with the diffusion coefficient. In particular, we expect a similar claim holds for a cubic nonlinearity, although we are not able to determine the size of the range quantitatively in that case.

Note that here we have an expression for $\gamma^{\prime}(0)$ when $A=0=\beta$ and when $A=\frac{1}{2}, \beta=-\frac{1}{2}$, but not for values of $A$ in $\left(0, \frac{1}{2}\right)$. We are interested in the quantity $\gamma^{\prime}(0)$ primarily when $a_{1}=a_{2}$. That is, if we consider $\gamma=\gamma(\alpha, A, \beta)$, we want to compute $\left.\frac{\partial \gamma}{\partial \alpha}\right|_{\left(\alpha, A, \beta^{*}\right)=\left(0, A, \beta^{*}\right)}$ for $A \in\left(0, \frac{1}{2}\right)$, where $\beta^{*}$ is such that $a_{1}=a_{2}$. The difficulty in extending the results of Theorem 5.1 to $A \in\left(0, \frac{1}{2}\right)$ lies in determining analytically the relationship between $A$ and $\beta^{*}$.

We now turn our attention to the proof of Theorem 5.1.

Proof. Let $\gamma_{i}=\gamma_{i}(\alpha)$ and let $W, X, Y, Z, F_{i, j}$, and $G_{i, j}$ be functions of $\alpha$ as well. We would like to calculate $\gamma_{i}^{\prime}(0)$. To do this note that

$$
W^{\prime}(0)=X^{\prime}(0)=Y^{\prime}(0)=Z^{\prime}(0)=Q^{\prime}(0)=0,
$$

and from this we obtain

$$
F_{i, \beta_{i}}^{\prime}(0)=F_{i, \beta_{i}+1}^{\prime}(0)=F_{i, \beta_{i}-1}^{\prime}(0)=G_{i, \beta_{i}}^{\prime}(0)=G_{i, \beta_{i}+1}^{\prime}(0)=G_{i, \beta_{i}-1}^{\prime}(0)=0
$$

and

$$
\begin{aligned}
F_{i, \beta^{*}}^{\prime}(0) & =-(1-A) R_{i^{*}} Y, \\
F_{i, \beta^{*}+1}^{\prime}(0) & =-A R_{i^{*}} Y, \\
G_{i, \beta^{*}}^{\prime}(0) & =-(1-A) R_{i^{*}} Z, \\
G_{i, \beta^{*}+1}^{\prime}(0) & =-A R_{i^{*}} Z .
\end{aligned}
$$

Since $D_{1}=D_{2}:=D$, we have $R_{1}=R_{2}:=R$, and

$$
\gamma_{i}^{\prime}(0)=\frac{2}{\pi p} \int_{0}^{p} \int_{0}^{\infty} \frac{T(s)}{\left(t^{2}+T^{2}(s)\right)^{2}} P(s) d t d s-\frac{1}{\pi} \int_{0}^{\infty} \frac{1}{s T^{2}(s)} Q(s) d s,
$$

where

$$
\begin{aligned}
& T(s)=2 D C(s)-1, \\
& P(s)=R(s)\left[(1-A) \cos \left(s\left(\beta_{i}-\beta_{i^{*}}\right)\right)+A \cos \left(s\left(\beta_{i}-\left(\beta_{i^{*}}+1\right)\right)\right)\right], \\
& Q(s)=R(s)\left[(1-A) \sin \left(s\left(\beta_{i}-\beta_{i^{*}}\right)\right)+A \sin \left(s\left(\beta_{i}-\left(\beta_{i^{*}}+1\right)\right)\right)\right] .
\end{aligned}
$$

Some calculation yields that the complex equation $T(z)=0$ has roots in

$$
\left\{z=a+i b \mid a=2 m \pi \text { for } m \in \mathbb{Z}, b=\cosh ^{-1}\left(1+\frac{1}{2 D}\right)\right\} .
$$

We focus on the calculation of the inner integral in the first term of (5.26). The complex roots of $\left.z^{2}+T^{2}(s)\right)^{2}=0$ are $z= \pm i T(s)$. Note that since $T(s)<0$ for real $s, P_{1}=-i T(s)$ lies in the upper half-plane. Denoting

$$
f(z)=\frac{1}{\left(z^{2}+T^{2}(s)\right)^{2}}=\frac{1}{(z-i T(s))^{2}(z+i T(s))^{2}},
$$

we have that

$$
\operatorname{res}_{f}\left(P_{1}\right)=\frac{i}{4 T^{3}(s)}
$$


Then

$$
\int_{0}^{\infty} \frac{T(s)}{\left(t^{2}+T^{2}(s)\right)^{2}} P(s) d t=\pi i \operatorname{res}_{f}\left(P_{1}\right)=\frac{-\pi}{4 T^{3}(s)}
$$

and

$$
\frac{2}{\pi p} \int_{0}^{p} \int_{0}^{\infty} \frac{T(s)}{\left(t^{2}+T^{2}(s)\right)^{2}} P(s) d t d s=-\frac{1}{2 p} \int_{0}^{p} \frac{1}{T^{2}(s)} P(s) d t:=\Psi
$$

Let

$$
p^{*}=\operatorname{lcm}\left(p_{i, j}^{*}\right), \text { and } q_{i, j}=\frac{p^{*}}{p_{i, j}^{*}} .
$$

To compute the integral $\Psi$, we use the change of variables $z=e^{\frac{i s}{p^{*}}}, d z=\frac{i}{p^{*}} e^{\frac{i s}{p^{*}}} d s=$ $\frac{i z}{p^{*}} d s$, which results in a contour integral around the unit circle. Now we may write

$$
\begin{aligned}
& T(z)=2 D\left(\frac{z^{p^{*}}+z^{-p^{*}}}{2}-1\right)-1, \\
& P(z)=2 D\left(\frac{z^{p^{*}}+z^{-p^{*}}}{2}-1\right)\left[(1-A)\left(\frac{z^{q_{i, \beta_{i}}}+z^{-q_{i, \beta_{i}}}}{2}\right)+A\left(\frac{z^{q_{i, \beta_{i} *+1}}+z^{-q_{i, \beta_{i}+1}}}{2}\right)\right] .
\end{aligned}
$$

Multiplying the top and bottom of the integrand by $z^{2 p^{*}-1}$ leaves the denominator as the square of a quadratic in $z^{p^{*}}$, with zeros given by

$$
z_{ \pm}^{p^{*}}=1+\frac{1}{2 D} \pm \frac{\sqrt{4 D+1}}{2 D}
$$

We have $\left|z_{-}^{p^{*}}\right|<1<\left|z_{+}^{p^{*}}\right|$, so the integrand has $p^{*}$ distinct poles inside the unit circle, each of order 2 . Labeling these poles $r_{1}, \ldots, r_{p^{*}}$ we have

$$
\Psi=-\frac{q}{i} \frac{1}{2 p} 2 \pi i \operatorname{res} \sum_{m=1}^{q}\left(\frac{P(z)}{T^{2}(z)}, r_{i}\right)=-\frac{1}{2} \operatorname{res} \sum_{m=1}^{q}\left(\frac{P(z)}{T^{2}(z)}, r_{i}\right) .
$$

This quantity can be computed explicitly in several special cases. We will start with $A=0, \beta=0$. In this case, $P(s)=R(s), p^{*}=1$, and $Q(s)=0$. Then we have

$$
r_{1}=1+\frac{1}{2 D}-\frac{\sqrt{4 D+1}}{2 D}
$$

and

$$
\operatorname{res}\left(\frac{P(z)}{T^{2}(z)}, r_{1}\right)=\frac{-2 D}{(4 D+1)^{\frac{3}{2}}},
$$

which gives us

$$
\gamma_{i}^{\prime}(0)=\frac{D}{(4 D+1)^{\frac{3}{2}}}>0 .
$$


For $A=\frac{1}{2}, \beta=-\frac{1}{2}$, we have $P(s)=R(s) \cos \left(\frac{s}{2}\right), p^{*}=2$, and $Q(s)=0$. We use the roots

$$
r_{1}, r_{2}= \pm \frac{\sqrt{2 D(2 D+1-\sqrt{4 D+1})}}{2 D}
$$

and

$$
\operatorname{res}\left(\frac{P(z)}{T^{2}(z)}, r_{1}\right)+\operatorname{res}\left(\frac{P(z)}{T^{2}(z)}, r_{2}\right)=0,
$$

implying $\gamma_{i}^{\prime}(0)=0$ in this case.

Unfortunately, explicit results like those given above are very difficult to find for more general parameter values. The problem lies in the computation of the second integral in equation (5.26). In an attempt to carry out this calculation, we see that the integral

$$
\Omega=\frac{1}{\pi} \int_{0}^{\infty} \frac{Q(z)}{z T^{2}(z)} d z
$$

can be evaluated using the calculus of residues. The poles of this integrand are given by

$$
r_{m}=2 m \pi+i \cosh ^{-1}\left(1+\frac{1}{2 D}\right)
$$

which are of order 2. Then

$$
\Omega=\pi i \sum_{m=-\infty}^{\infty} \operatorname{res}\left(\frac{Q(z)}{z T^{2}(z)}, r_{m}\right)
$$

Now using ' to denote differentiation with respect to the complex variable $z$, we write $(5.45)$

$$
\operatorname{res}\left(\frac{Q(z)}{z T^{2}(z)}, r_{m}\right)=-\frac{Q^{\prime}\left(r_{m}\right)}{r_{m}(4 D+1)}+\frac{Y\left(r_{m}\right)}{r_{m} D(4 D+1)}\left[\frac{1}{r_{m}}-\frac{i(2 D+1) \sqrt{4 D+1}}{4 D+1}\right] .
$$

Since $\beta_{i}-\beta_{i^{*}}$ may take on any rational value, it is very difficult to simplify, in general, the expressions for $Q^{\prime}\left(r_{m}\right)$ and $Y\left(r_{m}\right)$.

5.2. Numerical results on propagation failure. Figures 8 and 9 illustrate the effect of moving the coupling parameter $\alpha$ away from zero. Notice that when $A=0, \beta=0$, an increase in the coupling parameter $\alpha$ results in an increase in the range of propagation failure. However, when $A=.5, \beta=-.5$, this range decreases when $\alpha$ increases, suggesting that in the case of myelinated axons, nonalignment of the nodes of Ranvier allows for propagation in a larger range of values of the detuning parameter.

The plots in Figure 10 show the effects of coupling for positive wave speeds away from zero. Note that although our analytical expressions describe $a_{i}$ as a function of $c$, these plots allow us to see what happens to the wave speed if we fix a value for $a_{1}=a_{2}$ and then begin coupling. The results are especially interesting for small wave speeds: these plots indicate that coupling increases the wave speed for small wave 

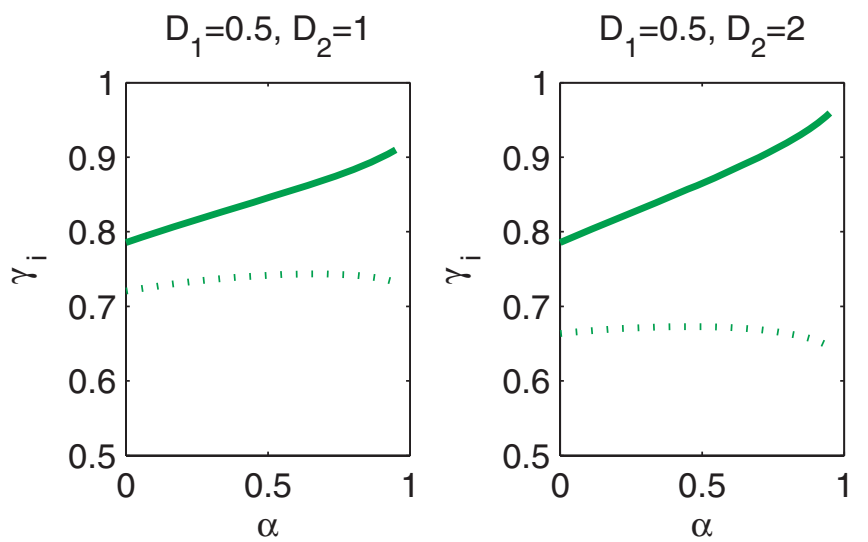

$$
\mathrm{D}_{1}=0.5, \mathrm{D}_{2}=1
$$

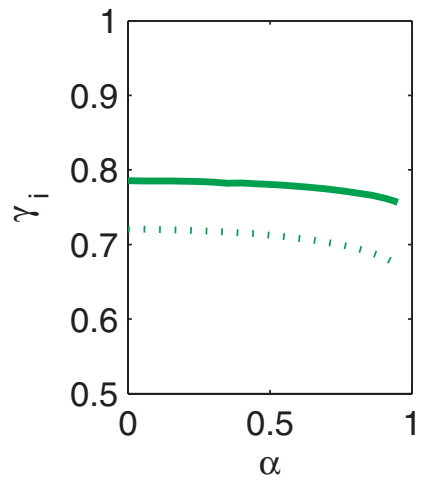

$D_{1}=0.5, D_{2}=2$

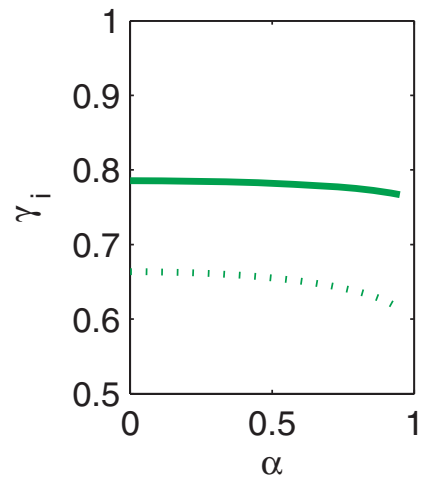

$D_{1}=1, D_{2}=2$

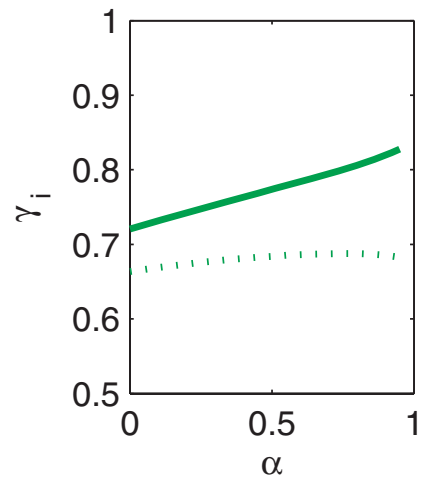

$D_{1}=1, D_{2}=2$

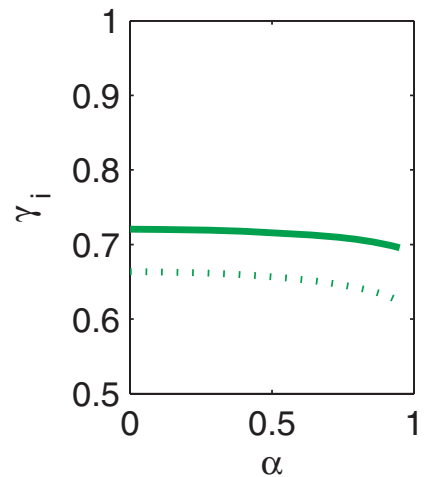

FIG. 8. In the top plots, $A=0=\beta$; in the bottom plots, $A=.5, \beta=-.5$. The plots show the range of propagation failure when $D_{1} \neq D_{2}$. The solid line represents $a_{1}$ and the dotted line $a_{2}$. (Note: values for failure were computed at $c=10^{-2}$.)

speeds when $A=.5$ and $\beta=-.5$ but that the same is not true when $A=0=\beta$. This also agrees with the results shown in Figure 9 when $D_{1}=D_{2}$, and the results obtained for $\left.\gamma_{i}^{\prime}(\alpha)\right|_{\alpha=0}$ in previous sections.

6. Conclusion. We have shown that traveling back solutions to our system exist (at least) for small values of the coupling coefficient $\alpha$, although further study is required to know the size of the range of $\alpha$ for which solutions exist. Using these solutions, we find that if the nodes are perfectly lined up or evenly staggered, and the waves travel together, then the sign of $g(c)=a_{1}-a_{2}$ remains unchanged for all $\alpha \in[0,1)$.

If we limit ourselves to the case $D_{1}=D_{2}$, we can comment on the questions we set out to answer: namely, what are the effects of nonalignment and ephaptic coupling on the propagation of action potentials? We find that (a) if the nodes are perfectly lined up and the waves travel together, then the introduction of ephaptic coupling increases the size of the range of propagation failure, and (b) if the nodes are evenly staggered and the waves travel together, then the introduction of ephaptic coupling decreases the the size of the range of propagation failure.

The first result, in particular, agrees in spirit with results obtained in [2] with the cubic nonlinearity. There the authors use numerical methods to show that larger 

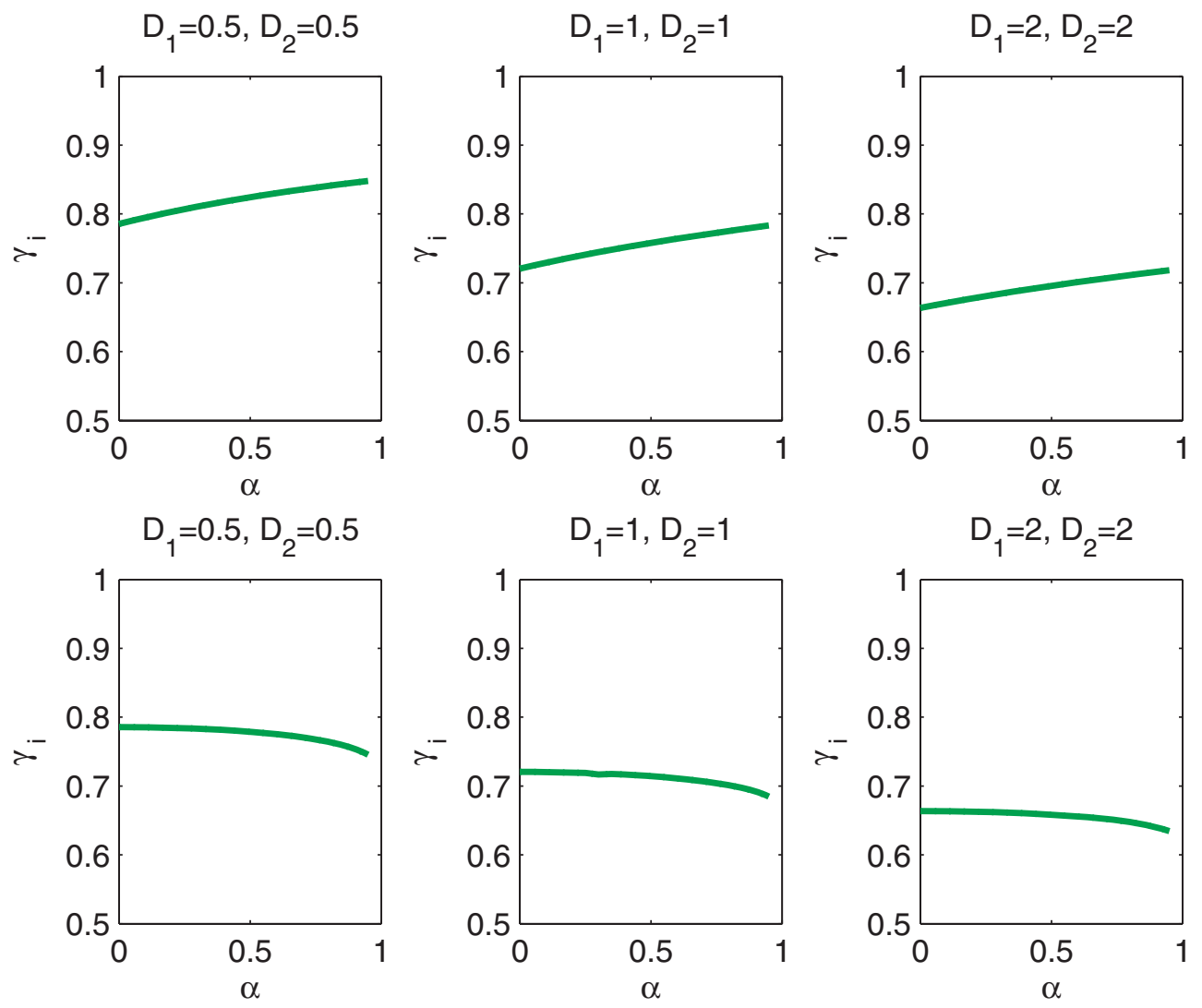

FIG. 9. In the top plots, $A=0=\beta$; in the bottom plots, $A=.5, \beta=-.5$. The plots show the range of propagation failure when $D_{1}=D_{2}$. In this case, $a_{1}=a_{2}$. Note how the ranges increase as $\alpha$ increases on the top but decrease as $\alpha$ increases on the bottom. (Note: values for failure were computed at $c=10^{-2}$.)

values of the diffusion coefficient are required to achieve a given wave speed (in particular, small wave speeds) as the coupling increases. They give similar results when the nodes are staggered, except that for small wave speeds, coupling appears to have little or no effect on the value of the diffusion coefficient required to achieve a certain wave speed. These results may not be compared directly to ours, however, since the results in [2] relate to the effect of coupling on the diffusion coefficient, whereas our results relate to the effect of coupling on the detuning parameters. In [20] and [21], evidence is given that ephaptic coupling can have a significant effect on the propagation of action potentials in a model for cardiac cells, but these results focus on the length of time required for an action potential on one fiber to affect the action potential on the other fiber, as opposed to the amount of time required for action potentials to proceed along a given fiber. The work [20,21] considers two types of coupling at once, ephaptic and ohmetric, and that approach may provide further insight into the dynamics of our present problem. The work in [3] is concerned with ephaptic coupling in nonmyelinated nerve fibers that might be modeled with (1.1) with large $D$. In [16], the authors focus on the effects of demyelination of nerve fibers in large bundles and observe a reduction in the speed of action potential propagation when the nodes of Ranvier are aligned. 

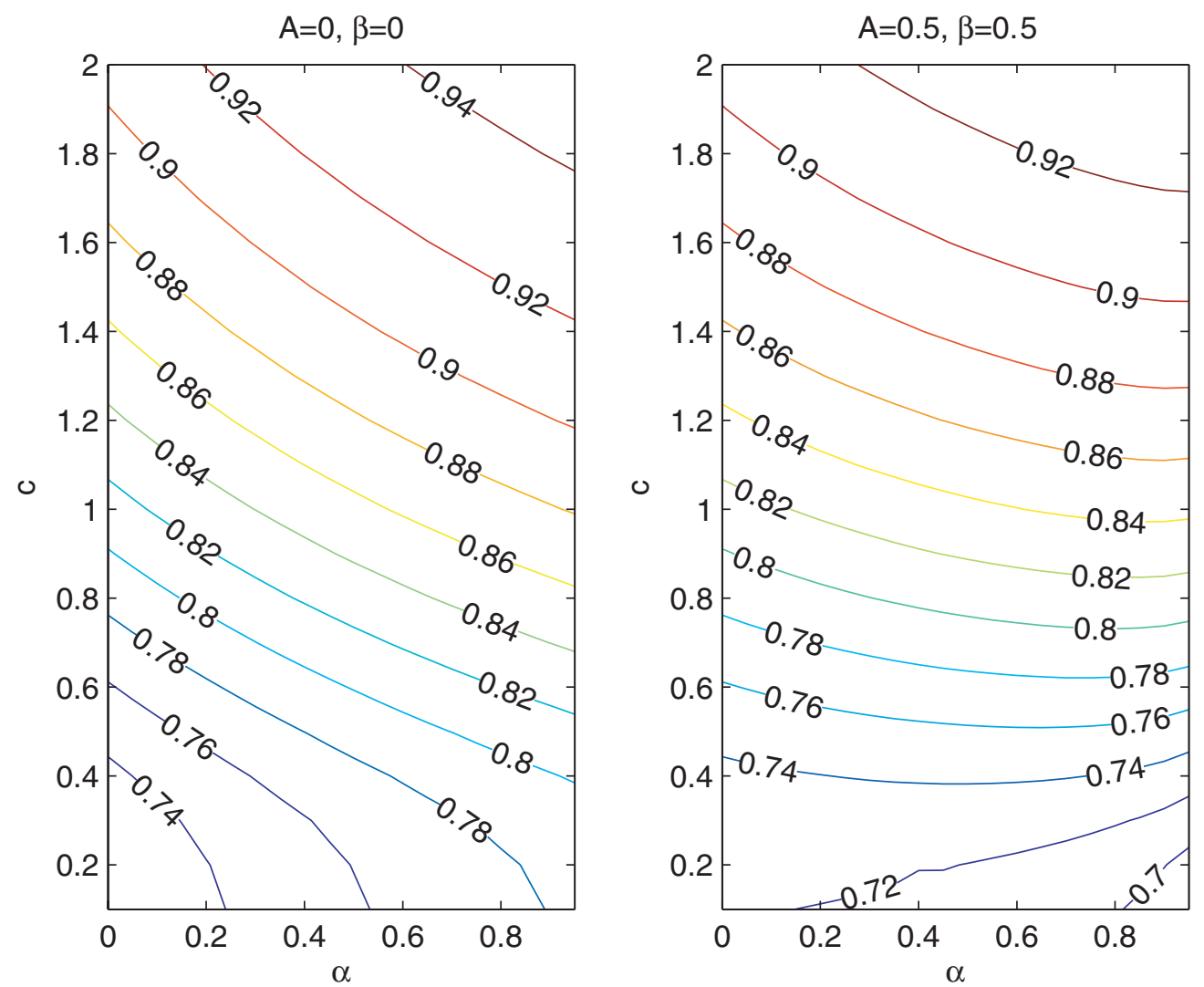

FIG. 10. Level curves of $a_{1}(\alpha, c)$ when $D_{1}=D_{2}=1$. Note that this implies $a_{1}=a_{2}$ for both plots. In the right plot, the value of $a_{i}(c)$ decreases with $\alpha$ for small $c$, whereas in the left plot, the value of $a_{i}(c)$ increases with $\alpha$ for small $c$. This is another illustration of the claim that coupling decreases the range of propagation failure when the nodes are staggered but increases the range when the nodes are aligned.

In addition, we have shown that for any value of $A$, there is a value of $\beta$ such that $a_{1}=a_{2}$. This means that regardless of the alignment of the nodes, we can have a situation in which the fibers are the same (i.e., $D_{1}=D_{2}$ and $a_{1}=a_{2}$ ) and the waves travel together. Perhaps a more surprising result is that with some parameter configurations, there are multiple such $\beta$ values. The existence of these multiple $\beta$ values gives rise to questions involving the stability of the solutions that we cannot answer with any certainty. The results in [2], and those in [1], point to the tendency of the two waves to match speeds (although in [1] a stronger type of coupling is considered). Given this fact, it seems reasonable that the same-speed solutions would be stable. However, the earlier results deal with waves that travel together at the same speed, and our results suggest the possibility of waves that travel at the same speed, with one wave lagging behind the other. This difference may give rise to interesting behavior with respect to the stability of the solutions.

Experience with the uncoupled case suggests that the piecewise linear and cubic problems may exhibit qualitatively similar behavior for small values of $c$ and small $(D \leq 1)$ values of $D$. It is reasonable to expect that qualitatively similar results related to propagation failure may hold in the cubic case, provided $D$ is not too large. 
7. Appendix A. We now present the details of the construction of our solution.

Lemma 7.1. Let $\left(\varphi_{1}, \varphi_{2}\right)$ be a solution to $(2.5),(2.7)$. Then there exists $\varepsilon_{0}>0$ such that for $i=1,2$,

$$
\left|\varphi_{i}(\xi)\right| \leq K e^{\varepsilon_{0} \xi} \text { for } \xi<\min \{-1, \beta\}
$$

for some $K>0$.

Proof. Let $\psi_{i}(\xi)=\varphi_{i}(-\xi)$, and note that from our boundary conditions, $\psi_{i}(\xi) \rightarrow$ 0 as $\xi \rightarrow \infty$. Then from the system (2.7), we have

$$
\begin{aligned}
c \psi_{1}^{\prime}(\xi)+\psi_{1}(\xi)=\frac{1}{1-\alpha^{2}}[ & D_{1}\left(L \psi_{1}\right)(\xi)-\alpha D_{2}\left(L \psi_{2}\right)(\xi) \\
- & \left.\alpha A\left(N^{*} \psi_{2}\right)(\xi)-\alpha^{2} A\left(N * \psi_{1}\right)(\xi+1)\right], \\
c \psi_{2}^{\prime}(\xi)+\psi_{2}(\xi)=\frac{1}{1-\alpha^{2}}[ & -\alpha D_{1}\left(L \psi_{1}\right)(\xi)+D_{2}\left(L \psi_{2}\right)(\xi) \\
+ & \left.\alpha^{2} A\left(N^{*} \psi_{2}\right)(\xi)+\alpha A\left(N^{*} \psi_{1}\right)(\xi+1)\right]
\end{aligned}
$$

for all $\xi>\max \{1,-\beta\}$, where

$$
\left(N^{*} \psi_{i}\right)(\xi)=c\left(\psi^{\prime}(\xi+1)-\psi^{\prime}(\xi)\right)+\psi(\xi+1)-\psi(\xi) .
$$

For this proof only, let $\hat{\psi}_{i}$ denote the Laplace transform

$$
\hat{\psi}_{i}(s)=\frac{1}{2 \pi} \int_{0}^{\infty} e^{-s \xi} \psi_{i}(\xi) d \xi .
$$

After applying the transform, we obtain the matrix equation

$$
M^{*}(s)\left[\begin{array}{c}
\hat{\psi}_{1}(s) \\
\hat{\psi}_{2}(s)
\end{array}\right]=J^{*}(s)\left[\begin{array}{c}
\psi_{1}(0) \\
\psi_{2}(0)
\end{array}\right]+K^{*}(s),
$$

where $M^{*}$ and $J^{*}$ are $2 \times 2$ matrix functions with entries

$$
\begin{aligned}
& M_{11}^{*}(s)=B^{*}(s)-2 k D_{1} C^{*}(s)+k \alpha^{2} A E(s) B(s), \\
& M_{12}^{*}(s)=2 k \alpha D_{2} C^{*}(s)-k \alpha A E(-s) B(s) \\
& M_{21}^{*}(s)=2 k \alpha D_{1} C^{*}(s)-k \alpha A E(s) B(s) \\
& M_{22}^{*}(s)=B^{*}(s)-2 k D_{2} C^{*}(s)+k \alpha^{2} A E(-s) B(s),
\end{aligned}
$$

and

$$
\begin{gathered}
J_{11}^{*}(s)=c\left(1+k \alpha^{2} A E^{*}(-s)\right), \\
J_{12}^{*}(s)=-c k \alpha A E^{*}(s), \\
J_{21}^{*}(s)=-c k \alpha A E^{*}(-s), \\
J_{22}^{*}(s)=c\left(1+k \alpha^{2} A E^{*}(s)\right), \\
k=\frac{1}{1-\alpha^{2}}, \\
C^{*}(s)=\cosh (s)-1, \\
B^{*}(s)=1+c s, \\
E^{*}(s)=1-e^{s},
\end{gathered}
$$


and $K^{*}$ is a vector with entries

$$
\begin{aligned}
& K_{1}^{*}(s)=k\left(D_{1} \Delta_{1}-\alpha D_{2} \Delta_{2}\right)+k \alpha A\left(\Omega_{2}+\alpha \Omega_{1}\right), \\
& K_{2}^{*}(s)=k\left(D_{2} \Delta_{2}-\alpha D_{1} \Delta_{1}\right)-k \alpha A\left(\Omega_{1}+\alpha \Omega_{1}\right),
\end{aligned}
$$

where

$$
\begin{aligned}
& \Delta_{i}=e^{-s} \int_{-1}^{0} e^{-s \xi} \psi_{i}(\xi) d \xi-e^{s} \int_{0}^{1} e^{-s \xi} \psi_{i}(\xi) d \xi \\
& \Omega_{i}= \pm e^{\mp s}\left(\int_{\mp 1}^{0} e^{-s \xi} \psi_{i}^{\prime}(\xi) d \xi+\int_{\mp 1}^{0} e^{-s \xi} \psi_{i}(\xi) d \xi\right) .
\end{aligned}
$$

Integrating by parts, we see that

$$
\int_{0}^{1} e^{-s \xi} \psi_{i}(\xi)=\left.\frac{-e^{-s \xi} \psi_{i}(\xi)}{s}\right|_{\xi=0} ^{1}+\frac{1}{s} \int_{0}^{1} e^{-s \xi} \psi_{i}^{\prime}(\xi) d \xi
$$

and

$$
\int_{0}^{1} e^{-s \xi} \psi_{i}^{\prime}(\xi)=\left.\frac{-e^{-s \xi} \psi_{i}^{\prime}(\xi)}{s}\right|_{\xi=0} ^{1}+\frac{1}{s} \int_{0}^{1} e^{-s \xi} \psi_{i}^{\prime \prime}(\xi) d \xi
$$

As long as these integrals are defined, we see that $K(s)=O\left(|s|^{-1}\right)$ as $|\operatorname{Im} s| \rightarrow \infty$, uniformly for Re $s$ bounded. Assuming $M^{*}$ is invertible, we obtain

$$
\left[\begin{array}{c}
\hat{\psi}_{1}(s) \\
\hat{\psi}_{2}(s)
\end{array}\right]=\left(M^{*}\right)^{-1} J^{*}(s)\left[\begin{array}{c}
\psi_{1}(0) \\
\psi_{2}(0)
\end{array}\right]+O\left(|s|^{-2}\right) \text { as } \quad|\operatorname{Im} s| \rightarrow \infty
$$

uniformly for Re $s$ bounded, since $\left(M^{*}\right)^{-1}=O\left(|s|^{-2}\right)$. This justifies the inversion of the Laplace transform, as well as the shift of contour around singularities on the imaginary axis. This gives us

$$
\begin{aligned}
\psi_{i}(\xi) & =\frac{1}{2 \pi i} \int_{\varepsilon_{0}-i \infty}^{\varepsilon_{0}+i \infty} e^{s \xi} \hat{\psi}_{i}(s) d s \\
& =\frac{1}{2 \pi i} \int_{-\varepsilon_{0}-i \infty}^{-\varepsilon_{0}+i \infty} e^{s \xi} \hat{\psi}_{i}(s) d s+\frac{1}{2 \pi i} \sum_{\operatorname{Re} s=0} e^{s \xi} \operatorname{res}\left(\hat{\psi}_{i}, s\right),
\end{aligned}
$$

where the first equality results from the Laplace inversion formula, and the second is a contour shift around any poles of $\hat{\psi}_{i}$ in the imaginary axis, and where $\varepsilon_{0}$ is small enough so that there are no poles of $\hat{\psi}_{i}$ for $-\varepsilon_{0} \leq \operatorname{Re} s<0$. Further, if

$$
\left(M^{*}\right)^{-1} J^{*}(s)\left[\begin{array}{l}
\psi_{1}(0) \\
\psi_{2}(0)
\end{array}\right]=O\left(|s|^{-1}\right) \text { as } \quad|\operatorname{Im} s| \rightarrow \infty
$$

for $\varepsilon<0$, then $\hat{\psi}_{i}=O\left(|s|^{-1}\right)$ as $|\operatorname{Im} s| \rightarrow \infty$.

We know that this condition is met, since $\left(M^{*}(s)\right)^{-1}=O\left(|s|^{-1}\right)$ and $J^{*}$ oscillates as $|\operatorname{Im} s| \rightarrow \infty$. Since we also have, from our boundary conditions, that $\psi_{i}(\xi) \rightarrow 0$ as $\xi \rightarrow \infty$, it is clear that

$$
\operatorname{res}\left(\hat{\psi}_{i}, s\right)=0 \text { for } \operatorname{Im} s=0 .
$$


This fact, together with (7.11) and (7.12), gives us that

$$
\psi_{i}(\xi) \mid \leq K e^{-\varepsilon_{0} \xi} \text { for } \xi>\max \{1,-\beta\}
$$

for $K>0$, which is equivalent to the claim made in the statement of the lemma.

Now define

$$
\varphi_{i, \varepsilon}(\xi)=e^{-\varepsilon \xi} \varphi_{i}(\xi),
$$

where $\varepsilon>0$ is sufficiently small. By Lemma 7.1, if $\left(\varphi_{1}, \varphi_{2}\right)$ satisfies $(2.5),(2.7)$, then $\varphi_{i, \varepsilon}(\xi) \rightarrow 0$ exponentially fast as $\xi \rightarrow+\infty$ and $\xi \rightarrow-\infty$.

After writing the system (2.7) in terms of the $\varphi_{i, \varepsilon}(\xi)$, we multiply both sides of each equation by appropriate factors, integrate over $\mathbb{R}$, and solve for the Fourier transforms of the $\varphi_{i, \varepsilon}$. Using the Fourier transform

$$
\hat{\varphi}_{i, \varepsilon}(s)=\int_{-\infty}^{+\infty} e^{-i s \xi} \varphi_{i, \varepsilon}(\xi) d \xi
$$

we obtain the matrix equation

$$
M(s-i \varepsilon)\left[\begin{array}{c}
\hat{\varphi}_{1, \varepsilon}(s) \\
\hat{\varphi}_{2, \varepsilon}(s)
\end{array}\right]=\frac{1}{i s+\varepsilon} N(s),
$$

where $M$ is a $2 \times 2$ matrix function with entries

$$
\begin{aligned}
& M_{11}(s)=B(s)-2 k D_{1} C(s)+k \alpha^{2} A E(s) B(s), \\
& M_{12}(s)=2 k \alpha D_{2} C(s)-k \alpha A E(-s) B(s), \\
& M_{21}(s)=2 k \alpha D_{1} C(s)-k \alpha A E(s) B(s), \\
& M_{22}(s)=B(s)-2 k D_{2} C(s)+k \alpha^{2} A E(-s) B(s),
\end{aligned}
$$

$N$ is a vector with entries

$$
\begin{aligned}
& N_{1}(s)=1-k \alpha A\left[E(-s) e^{-i s \beta}-\alpha E(s)\right] \\
& N_{2}(s)=e^{-i s \beta}-k \alpha A\left[E(s)-\alpha E(-s) e^{-i s \beta}\right],
\end{aligned}
$$

and

$$
\begin{aligned}
k & =\frac{1}{1-\alpha^{2}}, \\
C(s) & =\cos (s)-1, \\
B(s) & =1-i c s, \\
E(s) & =1-e^{i s} .
\end{aligned}
$$

The result given in Lemma 2.1 allows us to invert the matrix $M$, and we arrive at the solution of the matrix equation (7.18):

$$
\begin{aligned}
\hat{\varphi}_{1, \varepsilon}(s) & =\frac{M_{22}(s-i \varepsilon) N_{1}(s-i \varepsilon)-M_{12}(s-i \varepsilon) N_{2}(s-i \varepsilon)}{(i s+\varepsilon) \operatorname{det} M(s-i \varepsilon)} \\
\hat{\varphi}_{2, \varepsilon}(s) & =\frac{M_{11}(s-i \varepsilon) N_{2}(s-i \varepsilon)-M_{21}(s-i \varepsilon) N_{1}(s-i \varepsilon)}{(i s+\varepsilon) \operatorname{det} M(s-i \varepsilon)}
\end{aligned}
$$


Then using the Fourier inversion formula and the definition in (7.16), we have

$$
\begin{aligned}
& \varphi_{1}(\xi)=\frac{1}{2 \pi} \int_{-\infty}^{+\infty} e^{\xi(i s+\varepsilon)} \hat{\varphi}_{i, \varepsilon}(s) d s=\frac{1}{2 \pi i} \int_{-i \varepsilon-\infty}^{-i \varepsilon+\infty} \frac{e^{i s \xi}}{s} \frac{M_{22}(s) N_{1}(s)-M_{12}(s) N_{2}(s)}{\operatorname{det}(M(s))} \\
& \varphi_{2}(\xi)=\frac{1}{2 \pi} \int_{-\infty}^{+\infty} e^{\xi(i s+\varepsilon)} \hat{\varphi}_{i, \varepsilon}(s) d s=\frac{1}{2 \pi i} \int_{-i \varepsilon-\infty}^{-i \varepsilon+\infty} \frac{e^{i s \xi}}{s} \frac{M_{11}(s) N_{2}(s)-M_{21}(s) N_{1}(s)}{\operatorname{det}(M(s))} .
\end{aligned}
$$

Applying the Cauchy integral formula, we shift the contour of integration to the real axis, with a semicircle around the origin. That is, we rewrite the integral in the form

$$
\varphi_{1}(\xi)=\frac{1}{2 \pi i}\left(\int_{-\infty}^{-\varepsilon}+\int_{\varepsilon}^{\infty}+\int_{C_{\varepsilon}}\right) \frac{e^{i s \xi}}{s} \frac{M_{22}(s) N_{1}(s)-M_{12}(s) N_{2}(s)}{\operatorname{det}(M(s))},
$$

where $C_{\varepsilon}$ is lower semicircle of radius $\varepsilon$ around the origin. We may use a standard residue calculation for the third of these integrals, and the first two may be combined by using a change of variables in the first integral. After this, we use Euler's formula $\exp (i x)=\cos (x)+i \sin (x)$, and then we simplify by collecting sines and cosines according to their arguments. This leaves us with

$$
\varphi_{i}(\xi)=\frac{1}{2}+\frac{1}{2 \pi} \int_{0}^{\infty} \sum_{j \in M_{i}} F_{i, j}(s) \cos \left(s\left(\xi-\xi_{j}\right)\right)+G_{i, j}(s) \frac{1}{s} \sin \left(s\left(\xi-\xi_{j}\right)\right) d s
$$

where $F_{i, j}$ and $G_{i, j}$ are as in (2.9).

8. Appendix B. We now present the proof of Lemma 2.1.

Lemma 8.1. Let $\alpha \in[0,1), A \in[0,1), \beta \in \mathbb{R}$, and $c \neq 0$. Then

$$
|\operatorname{det} M(s)|^{2} \geq 1
$$

for $s \in \mathbb{R}$.

Proof. Using the definitions above, we may write $|\operatorname{det} M(s)|^{2}$ as

$$
|\operatorname{det} M(s)|^{2}=a^{2}+b^{2}=c^{4} s^{4}\left(b_{2}^{2}\right)+c^{2} s^{2}\left(2 b_{2}^{2}+b_{1}^{2}+2 b_{2}\left(b_{1}-b_{0}\right)\right)+\left(b_{2}+b_{1}+b_{0}\right)^{2} .
$$

Clearly $b_{2}^{2} \geq 0$ for $s \in \mathbb{R}$. Also,

$$
b_{2}+b_{1}+b_{0}=1-2 k C(s)\left[D_{1}+D_{2}-2 D_{1} D_{2} C(s)+\alpha^{2} A(1-A)\right] \geq 1
$$

since $C(s) \leq 0$. It remains to show that the coefficient of $c^{2} s^{2}$ is nonnegative. Sorting by powers of $C(s)$, we have

$$
\begin{aligned}
(8.4) 2 b_{2}^{2}+b_{1}^{2}+2 b_{2}\left(b_{1}-b_{0}\right)= & C^{3}(s)\left[16 k^{2} D_{1} D_{2} \alpha^{2} A(1-A)\right] \\
& +C^{2}(s) 4 k\left(\left[k \left[2 \alpha^{2} A(1-A)\left(D_{1}+D_{2}+\alpha^{2} A(1-A)\right)\right.\right.\right. \\
& \left.\left.\left.\quad+\left(D_{1}+D_{2}\right)^{2}\right]-2 D_{1} D_{2}\right]\right) \\
& +C(s) 4 k\left[-2 \alpha^{2} A(1-a)-\left(D_{1}+D_{2}\right)\right] \\
& +2
\end{aligned}
$$


from which we see that the coefficient of $C^{0}(s)$ is positive and that of $C^{1}(s)$ is negative, as desired. It is convenient to express the order 2 and order 3 terms as

$$
\begin{array}{r}
C^{2}(s)\left(4 k^{2}\left[2 \alpha^{2} A(1-A)\left(D_{1}+D_{2}+\alpha^{2} A(1-A)\right)\right]\right) \\
+C^{2}(s)\left(4 k\left[\left(D_{1}+D_{2}\right)^{2} k-2 k D_{1} D_{2}+C(s) 4 k D_{1} D_{2} \alpha^{2} A(1-A)\right]\right),
\end{array}
$$

where once again the terms on the top row are nonnegative. Finally, we have the estimate for the coefficient on the bottom

$$
\begin{aligned}
& k\left[D_{1}^{2}+D_{2}^{2}+4 k D_{1} D_{2} C(s) \alpha^{2} A(1-A)\right]+2 D_{1} D_{2}(k-1) \\
\geq & k\left[D_{1}^{2}+D_{2}^{2}+4 k D_{1} D_{2} C(s) \alpha^{2} A(1-A)\right] \\
\geq & D_{1}^{2}+D_{2}^{2}-2 D_{1} D_{2} \geq 0
\end{aligned}
$$

since $-2 \leq C(s) \leq 0, \alpha<1$, and $4 A(1-A) \leq 1$ for $A \in[0,1)$. Hence the coefficient of $c^{2} s^{2}$ in $|\operatorname{det} M(s)|^{2}$ is greater than or equal to two. This estimate gives us the result stated above.

9. Appendix C. We now prove the claim (5.8) by showing that $\sum_{n=0}^{\infty} E_{n} \rightarrow 0$ as $c \rightarrow 0$. Recall

$$
\begin{aligned}
\left|E_{n}\right| & =\left|\int_{n p}^{(n+1) p} F_{i, j}(s) \cos \left(s\left(\beta_{i}-j\right)\right) d s-\int_{n p}^{(n+1) p} F_{i, j}(n p, s) \cos \left(s\left(\beta_{i}-\xi_{j}\right)\right) d s\right| \\
& \leq\left|\int_{n p}^{(n+1) p}\left[F_{i, j}(s)-F_{i, j}(n p, s)\right] d s\right| .
\end{aligned}
$$

Also, since $\left|R_{i}(s)\right| \leq 2$ and all other terms in the $F_{i, j}$ are constant, it will suffice to look at

$$
\left|\int_{n p}^{(n+1) p}[W(s)-W(n p, s)] d s\right| \text { and }\left|\int_{n p}^{(n+1) p}[Y(s)-Y(n p, s)] d s\right| .
$$

The following applies to both cases. First, make a change of variables $s \rightarrow \frac{s}{c}$ in both terms of the difference. Also, the differences $W(s)-W(n p, s)$ and $Y(s)-Y(n p, s)$ may be expressed in the form

$$
\frac{\gamma_{2}(s) s^{2}+\gamma_{0}(s)}{\delta_{4}(s) s^{4}+\delta_{2}(s) s^{2}+\delta_{0}(s)}-\frac{\gamma_{2}(s)(n p c)^{2}+\gamma_{0}(s)}{\delta_{4}(s)(n p c)^{4}+\delta_{2}(s)(n p c)^{2}+\delta_{0}(s)}
$$

where the $\gamma$ and $\delta$ are functions of $s$, but we will suppress this dependence to make the notation lighter. Then for $n \geq 1$, 
$(9.4)$

$$
\begin{aligned}
\left|E_{n}\right| & \leq \int_{n p c}^{(n+1) p c}\left((n p c)^{2}-s^{2}\right) \frac{\gamma_{2} \delta_{4}(n p c)^{2} s^{2}+\gamma_{0} \delta_{4}\left((n p c)^{2}+s^{2}\right)+\gamma_{0} \delta_{2}-\gamma_{2} \delta_{0}}{\left(\delta_{4} s^{4}+\delta_{2} s^{2}+\delta_{0}\right)\left(\delta_{4}(n p c)^{4}+\delta_{2}(n p c)^{2}+\delta_{0}\right)} d s \\
& \leq \int_{n p c}^{(n+1) p c}(2 n+1)(p c)^{2} \frac{\gamma_{2} \delta_{4}(n p c)^{2} s^{2}+\gamma_{0} \delta_{4}\left((n p c)^{2}+s^{2}\right)+\gamma_{0} \delta_{2}-\gamma_{2} \delta_{0}}{\left(\delta_{4} s^{4}+\delta_{2} s^{2}+\delta_{0}\right)\left(\delta_{4}(n p c)^{4}+\delta_{2}(n p c)^{2}+\delta_{0}\right)} d s \\
& \leq \int_{n p c}^{(n+1) p c}(2 n+1)(p c)^{2} \frac{\gamma_{2} \delta_{4}(n p c)^{2} s^{2}+\gamma_{0} \delta_{4}\left((n p c)^{2}+s^{2}\right)+\gamma_{0} \delta_{2}-\gamma_{2} \delta_{0}}{\left(\delta_{4} s^{4}+\delta_{2} s^{2}+\delta_{0}\right)\left(\frac{\delta_{4}}{16} s^{4}+\frac{\delta_{2}}{4} s^{2}+\delta_{0}\right)} d s \\
& \leq 16 \int_{n p c}^{(n+1) p c}(2 n+1)(p c)^{2} \frac{\gamma_{2} \delta_{4}(n p c)^{2} s^{2}+\gamma_{0} \delta_{4}\left((n p c)^{2}+s^{2}\right)+\gamma_{0} \delta_{2}-\gamma_{2} \delta_{0}}{\left(s^{2}+1\right)^{4}} d s \\
& \leq 16 \int_{n p c}^{(n+1) p c}(2 n+1)(p c)^{2} \frac{\gamma_{2} \delta_{4} s^{4}+2 \gamma_{0} \delta_{4} s^{2}+\gamma_{0} \delta_{2}-\gamma_{2} \delta_{0}}{\left(s^{2}+1\right)^{4}} d s \\
& \leq 16 \int_{n p c}^{(n+1) p c} 3 s p c \frac{\gamma_{2} \delta_{4} s^{4}+2 \gamma_{0} \delta_{4} s^{2}+\gamma_{0} \delta_{2}-\gamma_{2} \delta_{0}}{\left(s^{2}+1\right)^{4}} d s \\
& \leq 48 p c \int_{n p c}^{(n+1) p c} K s \frac{s^{4}+2 s^{2}+1}{\left(s^{2}+1\right)^{4}} d s \\
& \leq 48 p c K \int_{n p c}^{(n+1) p c} \frac{s}{\left(s^{2}+1\right)^{2}} d s,
\end{aligned}
$$

where $K$ is a constant. Similarly,

$$
\begin{aligned}
\left|E_{0}\right| & \leq(p c)^{2} \int_{0}^{p c} \frac{\gamma_{0} \delta_{4}(p c)^{2}+\gamma_{0} \delta_{2}-\gamma_{2} \delta_{0}}{\left(\delta_{4} s^{4}+\delta_{2} s^{2}+\delta_{0}\right) \delta_{0}} d s \\
& \leq K^{\prime}(p c)^{2} \int_{0}^{p c} \frac{1}{\left(s^{1}+1\right)^{2}} d s \rightarrow 0 \quad \text { as } \quad c \rightarrow 0
\end{aligned}
$$

where $K^{\prime}$ is a constant. Using these estimates, we see that

$$
\sum_{n=0}^{\infty}\left|E_{n}\right| \leq\left|E_{0}\right|+48 p c K \int_{p c}^{\infty} \frac{s}{\left(s^{2}+1\right)^{2}} d s \rightarrow 0 \quad \text { as } \quad c \rightarrow 0,
$$

which justifies our claim in (5.8).

Acknowledgments. The authors are grateful to Chris Elmer and Weishi Liu for discussions related to this work and to the referees for helpful comments on an earlier version of this paper.

\section{REFERENCES}

[1] J. M. Bilbault, V. B. Kazantsev, P. Marquie, S. Morfu, and V. I. Nekorkin, Theoretical and experimental study of two discrete coupled Nagumo chains, Phys. Rev. E, 64 (2001), 036602 .

[2] S. Binczak, J. C. Eilbeck, and A. C. Scott, Ephaptic coupling of myelinated nerve fibers, Phys. D, 148 (2001), pp. 159-174.

[3] H. Bokil, N. LaAris, K. Blinder, M. Ennis, and A. Keller, Ephaptic interactions in the mammalian olfactory system, J. Neurosci., 2001, 21:RC173(1-5).

[4] A. Bose, Symmetric and antisymmetric pulses in parallel coupled nerve fibres, SIAM J. Appl. Math., 55 (1995), pp. 1650-1674. 
[5] A. Bose And C. K. R. T. Jones, Stability of the in-phase travelling wave solution in a pair of coupled nerve fibers, Indiana Univ. Math. J., 44 (1995), pp. 189-220.

[6] J. W. Cahn, J. Mallet-Paret, And E. S. VAn Vleck, Traveling wave solutions for systems of ODEs on a two-dimensional spatial lattice, SIAM J. Appl. Math., 59 (1998), pp. 455-493.

[7] S. N. Chow, J. Mallet-Paret, W. Shen, Traveling waves in lattice dynamical systems, J. Differential Equations, 149 (1998), pp. 248-291.

[8] C. E. Elmer and E. S. VAn Vleck, Traveling wave solutions for bistable differential-difference equations with periodic diffusion, SIAM J. Appl. Math., 61 (2001), pp. 1648-1679.

[9] C. E. Elmer and E. S. Van Vleck, Spatially discrete Fitzhugh-Nagumo equations, SIAM J. Appl. Math., 65 (2005), pp. 1153-1174.

[10] T. ERneux And G. Nicolis, Propagating waves in discrete bistable reaction-diffusion systems, Phys. D, 67 (1993), pp. 237-244.

[11] G. FATH, Propagation failure of traveling waves in discrete bistable medium, Phys. D, 116 (1998), pp. 176-190.

[12] J. A. FEROE, Existence and stability of multiple impulse solutions of a nerve equation, SIAM J. Appl. Math., 42 (1982), pp. 235-246.

[13] J. P. KeENER, Frequency dependent decoupling of parallel excitable fibers, SIAM J. Appl. Math., 49 (1989), pp. 210-230.

[14] R. S. Mackay and J.-A. Sepulchre, Multistability in networks of weakly coupled bistable units, Phys. D, 82 (1995), pp. 243-254.

[15] H. McKean, Nagumo's Equation, Adv. Math., 4 (1970), pp. 209-223.

[16] S. Reutskiy, E. Rossoni, And B. Tirozzi, Conduction in bundles of demyelinated nerve fibers: Computer simulation, Biol. Cybern., 89 (2003), pp. 439-448.

[17] J. Rinzel and J. B. Keller, Traveling wave solutions of a nerve conduction equation, Biophys. J., 13 (1973), pp. 1313-1337.

[18] A. Scott, Neuroscience: A Mathematical Primer, Springer, New York, 2002.

[19] L. F. Shampine, R. C. Allen, S. Pruess, Fundamentals of Numerical Computing, Wiley, New York, 1997.

[20] N. SPERElakis, Combined electric field and gap junctions on propagation of action potentials in cardiac muscle and smooth muscle in PSpice simulation, J. Electrocard., 36 (2003), pp. 279-293.

[21] N. SPERELAKIS, An electric field mechanism for transmission of excitation between myocardial cells, Circulation Res., 91 (2002), pp. 985-987.

[22] W.-P. WANG, Multiple impulse solutions to McKean's caricature of the nerve equation. I Existence, Comm. Pure Appl. Math., 41 (1988), pp. 71-103.

[23] W.-P. WANG, Multiple impulse solutions to McKean's caricature of the nerve equation. II Stability, Comm. Pure Appl. Math., 41 (1988), pp. 997-1025. 